\title{
An Improved Robust Adaptive Controller for a Fed-Batch Bioreactor with Input Saturation and Unknown Varying Control Gain via Dead-Zone Quadratic Forms
}

\author{
Alejandro Rincón ${ }^{1,2, *(\mathbb{C})}$, Gloria María Restrepo ${ }^{2}$ (D) and Óscar J. Sánchez ${ }^{3}[$ \\ 1 Grupo de Investigación en Desarrollos Tecnológicos y Ambientales-GIDTA, Facultad de Ingeniería y \\ Arquitectura, Universidad Católica de Manizales, Carrera 23 N. 60-63, Manizales 170002, Colombia \\ 2 Grupo de Investigación en Microbiología y Biotecnología Agroindustrial-GIMIBAG, Instituto de \\ Investigación en Microbiología y Biotecnología Agroindustrial, Facultad de Ciencias de la Salud, \\ Universidad Católica de Manizales, Carrera 23 N. 60-63, Manizales 170002, Colombia; grestrepo@ucm.edu.co \\ 3 Grupo de Investigación en Alimentos y Agroindustria, Departamento de Ingeniería, Universidad de Caldas, \\ Calle 65 N. 26-10, Manizales 170004, Colombia; osanchez@ucaldas.edu.co \\ * Correspondence: arincons@ucm.edu.co; Tel.: +57-(6)-8933050
}

Citation: Rincón, A.; Restrepo, G.M.; Sánchez, Ó.J. An Improved Robust Adaptive Controller for a Fed-Batch Bioreactor with Input Saturation and Unknown Varying Control Gain via Dead-Zone Quadratic Forms.

Computation 2021, 9, 100. https:// doi.org/10.3390/computation9090100

Academic Editor: Demos T. Tsahalis

Received: 6 August 2021

Accepted: 10 September 2021

Published: 16 September 2021

Publisher's Note: MDPI stays neutral with regard to jurisdictional claims in published maps and institutional affiliations.

Copyright: () 2021 by the authors. Licensee MDPI, Basel, Switzerland. This article is an open access article distributed under the terms and conditions of the Creative Commons Attribution (CC BY) license (https:// creativecommons.org/licenses/by/ $4.0 /)$.

\begin{abstract}
In this work, a new adaptive controller is designed for substrate control of a fed-batch bioreactor in the presence of input saturation and unknown varying control gain with unknown upper and lower bounds. The output measurement noise and the unknown varying nature of reaction rate and biomass concentration and water volume are also handled. The design is based on dead zone quadratic forms. The designed controller ensures the convergence of the modified tracking error and the boundedness of the updated parameters. As the first distinctive feature, a new robust adaptive auxiliary system is proposed in order to tackle input saturation and control gain uncertainty. As the second distinctive feature, the modified tracking error converges to a compact region whose bound is user-defined, in contrast to related studies where the convergence region depends on upper bounds of either external disturbances, system states, model parameters or terms and model parameter values. Simulations confirm the properties of the closed loop behavior.
\end{abstract}

Keywords: adaptive control; augmented error signal; fed-batch bioreactor; input saturation; Lyapunov-like function

\section{Introduction}

Fed-batch bioreactors allow obtaining high amounts of biomass or products for a given process time. In turn, this is related to high growth rates or production rates [1,2]. The application of automatic control makes possible to have enhanced productivity during the process cultivation. To this end, substrate can be regulated at a value that leads to high growth rate in order to avoid the catabolic repression of the microorganisms [1,2].

Control stabilisation of bioreactors by using PI-like or non-adaptive controllers is hampered by system uncertainty, mainly lack of knowledge on: structure and coefficients of the reaction rates, reaction yields, concentration of products, and inflow substrate concentration. The main uncertainty comes from the reaction rate, as its coefficients usually vary with time. In addition, the reaction yield usually varies with time as well, and there could be measurement errors for the inflow substrate concentration, and substrate and biomass concentrations [3-6]. Controlling substrate and product in fed-batch bioreactors is also hampered by these challenges [7-10]. Nonlinear and adaptive control are capable of dealing with these uncertainties, while ensuring the asymptotic convergence of the tracking error. In addition, they can handle uncertainty on biomass concentration [11-14]. Regarding this, adaptive control of continuous bioreactors is addressed in [14,15], nonlinear control design for fed-batch processes is addressed in $[1,2,9,10,16]$, and adaptive control for fed-batch processes in [17]. 
In [16] a Lyapunov-based controller is designed for a fed-batch biomass culture. It is considered that the concentration of substrate and biomass is bounded upon bounded dilution rate. The controlled output is chosen as the substrate concentration, and the desired output is chosen as the value of the substrate concentration that leads to maximum product gaseous outflow rate. The Lyapunov function $(W)$ is defined as a quadratic function of the output tracking error $(e)$, and the chosen control law is of sliding type, such that the time derivative $(d W / d t)$ leads to boundedness of $W$ and $e$ and the convergence of $e$ to zero. However, the controller design disregards the unknown time varying nature of the control gain, the measurement noises in substrate concentration and culture volume, and also the effect of input saturation. In [17], the so-called decoupled adaptive controller (DAC) is applied for the culture of $P$. pastoris yeast. The substrate feed rate and the air flow rate are the manipulated variables, whereas the substrate and dissolved oxygen concentrations are the controlled outputs. The reaction rate terms are unknown. The controller is a combintaion of DAC and input-output linearizing control (DIOLC). The used DAC scheme consists of an application of model reference adaptive control (MRAC), and comprises updated parameters in order to provide estimates of the uncertain reaction rate terms. The matrix inversion principle is used to handle the combined effect of the two inputs. The controller was implemented on line, with adequate but improvable performance. However, the control gains are considered as accurately known in the control design, whereas the measurement noise in the state variables and the effect of input saturation are not taken into account by the control design. In [1], an extremum seeking control scheme is proposed for optimization of the specific growth rate in fed batch processes whose kinetics are substrate inhibited. The dilution rate is the manipulated variable, whereas the gradient of the specific growth rate is the controlled variable. The gradient of the specific growth rate is estimated through a high-order sliding mode observer, whereas the specific growth rate is estimated through an exponential observer, and the substrate concentration is estimated through an asymptotic observer, using the knowledge on the substrate to biomass yield and concentrations of biomass and feed substrate. The controller design is based on a high-order sliding mode scheme, using the estimates provided by the observer and the known yield coefficient, but not the kinetic model parameters. The stability of the closed loop variables is determined through Lyapunov functions. The controller was tested by simulation, considering measurement noise, and a time varying optimum of the specific growth rate. However, the uncertainty on the yield coefficient and the measurement noise in biomass and inflow substrate concentrations and the effect of input saturation are disregarded in the controller design. In [15], a robust adaptive controller is designed for a class of continuous wastewater treatment processes. It is assumed that: bacterial growth rates and inlet pollutant concentrations are unknown, but some upper and lower bounds are known; the biomass and substrate concentrations are unknown. The output is defined as the sum of the concentrations of the two substrates, whereas the manipulated input is defined as the dilution rate. The controller design is based on Lyapunov function, it uses the estimates of biomass concentration provided by the interval observer, and the estimates of reaction rates provided by the estimator. However, input saturation is not taken into account in the control design. In [14], a robust adaptive controller is designed for continuous bioreactors. The biomass concentration, the biomass growth rate, and the inflow substrate concentration are considered unknown to the controller, whereas the gas production is considered known. The substrate concentration is chosen as controlled output, and the dilution rate is chosen as manipulated input. The lack of knowledge on the inflow substrate concentration implies unknown control gain. The controller design is based on Lyapunov function, and uses an arrangement of the substrate uptake rate in terms of the measured gas production in order to partially tackle the uncertainty in the biomass growth rate. Moreover, parameter updating is used in order to cope with the lack of knowledge on the control gain and yield coefficient. However, the effect of input saturation is not taken into account in the control design. In [2], a controller is designed for a fed-batch process with dual substrate feeding and additive kinetics. The specific growth 
rate is the output to be controlled, and the feeding flow rates are the manipulated inputs. The biomass concentration, the working volume, the inflow substrate concentrations, and the yields of substrate to biomass are assumed known, whereas the specific growth rate is assumed unknown. The specific growth rate is estimated through a super twisting sliding mode observer, based on the known biomass concentration. The control law is proportional to biomass, and it achieves convergence of the tracking error. However, the control gain is considered accurately known and the effect of input saturation is not taken into account in the control design.

In addition to the effects of model uncertainty, closed loop performance can be severely affected by actuator saturation if it is unaccounted in the controller design, and considerable overshoot may occur [18-23]. In case of adaptive controllers, the stability of the closed loop system may be lost, and windup phenomenon may occur, in which adapted parameters change excessively. One possible solution is to stop adaptation during saturation moments, although this is not an optimal one $[19,24]$. One strategy used in control design for nonlinear systems with input saturation is the so-called augmented error signal (AES). It allows preserving closed loop stability and its effectiveness has been demonstrated in several mechanical systems. In adaptive controllers with AES strategy, excessive increase of updated parameters is prevented. The AES strategy is characterized by the fact that the regular tracking error is modified by adding the state of an auxiliary system which is a filter in terms of $\Delta u$, the difference between the constrained and unconstrained inputs $[19,24,25]$.

A pioneering incorporation of the AES strategy to Lyapunov-based adaptive control is the direct learning control scheme presented in [24]. Therein, it is proved that the closed loop signals are bounded despite input saturation, so that excessive increase of updated parameters is avoided. In addition, the modified tracking error asymptotically converges to a compact set of small size. In case that the control gain consists of the product of an unknown constant and a known function, the uncertainty of the constant is tackled by using an updated parameter, which is used in the auxiliary system [24]. The design procedure of the AES strategy of [24] comprises: (i) Formulation of an auxiliary system, involving the input error $\Delta u$, which is the error between the constrained and unconstrained input values; (ii) use of the modified tracking error as closed loop state variable instead of the regular tracking error, the modified tracking error consisting of the regular tracking error minus the state of the auxiliary system. The main features of the resulting closed loop system and controller are: (i) The control and update laws are function of the modified tracking error instead of the regular tracking error; (ii) the modified tracking error, the regular tracking error, and the parameter updating error are bounded despite input saturation; (iii) the modified tracking error asymptotically converges to a residual set of small size. Further control designs for input constrained systems use this AES adaptive control strategy, and the closed loop system and the controller commonly exhibit the above features, see $[19,26,27]$. Furthermore, these AES adaptive control designs incorporate other strategies, for instance: (i) Recursive backstepping approach, for handling high-order nonlinear systems (see $[19,25,26]$ ); (ii) fuzzy and neural network, for approximating the unknown system nonlinearities (see [19,26-29]); and (iii) state observers, for handling the lack of knowledge on unknown states (see [25,28,29]). In [27], an output feedback dynamic surface control is designed for an input constrained system subject to actuator failures and saturation. The unknown states are estimated through a state observer. The uncertain nonlinear terms are identified and approximated online through a Takagi-Sugeno (T-S) fuzzy system. The "explosion of complexity" is tackled via dynamic surface control (DSC) strategy. The effect of input saturation is tackled via AES strategy with auxiliary system. An updated parameter is used in order to tackle the lack of knownledge on an unknown positive constant that is the maximum value among the auxiliary signal, the desired output, the upper bound of the external disturbance, and the approximation error. However, the control gain is considered as accurately known in the control design, and the convergence region of the modified tracking error depends on the bounds of: external disturbances, system states, and model coefficients. Therefore, to achieve the convergence of the modified 
tracking error to a compact set of small user-defined size, such bounds must be known. Current AES robust adaptive control designs exhibit these two limitations (see also [19,25]).

To the authors knowledge, application of AES adaptive control to bioreactors and to systems with unknown varying control gain is overly scarce; some examples are mentioned as what follows in which other strategies as state observers and neural networks are incorporated in the controller. In [25], a general nonlinear time-delay system of $n$ differential equations is considered, and a recursive output feedback backstepping is used. Moreover, the model of a two-stage chemical reactor with recirculation is considered as a particular case of the general system. The reactor model corresponds to a second-order nonlinear system, where the first state is the known output, while the remaining states are unknown. The limitation is that the controller design considers the control gain as constant and perfectly known. In [29], a CSTR is considered, whose model corresponds to a secondorder nonlinear system, where the first state is the known output, whereas the remaining states are unknown. The controller uses neural network and a high-order sliding mode (HOSM) observer. An antiwindup compensator is used, which amounts to the auxiliary system of the AES strategy. The limitation is that the control gain is considered perfectly known. In [30], a SISO nonlinear system of $n$ order with unknown nonlinear control gain is considered. The unknown nonlinear nature of the control gain is tackled by using the Nussbaum function strategy. The main limitation is that the size of the convergence region of the modified tracking error depends on the unknown model coefficients and bounds.

In this study, a Lyapunov-based adaptive controller is developed for a fed-batch bioreactor in the presence of input saturation, unknown varying model parameters, output measurement noise, and unknown varying control gain, with unknown upper and lower bounds. An adaptive robust control design based on dead-zone quadratic forms is proposed for tackling the lack of knowledge on model parameters, control gain, and measurement noise. An improved robust auxiliary system is proposed for tackling the effect of input saturation, subject to varying control gain, with unknown upper and lower bounds. The stability analysis of the system under the formulated controller includes the following proofs: boundedness of the parameter updating error; convergence of the modified tracking error to a compact set of user-defined size; and convergence of the regular tracking error to a residual set of user-defined size, in the case that the input saturation eventually ceases. The main contributions of this paper with respect to closely related studies that consider input saturation are:

- A new auxiliary system is proposed, which is robust against varying and unknown control gain, and unknown upper and lower bounds. In contrast, in common adaptive control strategies for input saturation, the control gain is considered as constant and known in the definition of the auxiliary system (see [25,29]).

- The modified tracking error converges to a compact set whose width is user-defined, so that model coefficients, and bounds of either external disturbances, system states, model terms, and model coefficients are not required to be known. This is in contrast to current adaptive control designs for input saturation (see $[25,29,30])$, where the width of the convergence region depends on the aforementioned bounds.

- $\quad$ The noisy measurements of the substrate and volume are considered in the control design; the imperfectly known control gain is used instead of considering the control gain as completely unknown.

- $\quad$ The asymptotic convergence of the modified error is proved by means of the Barbalat's lemma, and it takes into account the formulated controller, which comprises the control and update laws and the auxiliary system, involving a saturation function.

The developed strategy for tackling the unknown varying control gain is a major contribution to the AES adaptive control literature.

The organization of the work is as follows. Section 2 presents: (i) the mass balance model of the system and the assumptions, including the measurement model; (ii) the features of the control gain, including boundedness features and their proofs. Section 3 presents the reference model and the statement of the control goal. Section 4 presents the 
adaptive controller design and the determination of the boundedness and convergence properties of the closed loop state variables. In Section 5, simulations results are presented. In Section 6, the conclusions are drawn.

The system model and assumptions presented in Section 2, and the reference model presented in Section 3, are taken from previous works. The proofs of the boundedness features of the control gain presented in Section 2 are original work of the authors, developed on the basis of the features of the state variables and model terms stated in the assumptions. The statement of the control goal presented in Section 3 mentions the convergence of the tracking error, the model and its assumptions, and some expected convergence features of the closed loop system. This is in agreement with robust control studies where the control goal statement comprises the convergence of the tracking error and in some cases also includes some challenges of the system and expected convergence features. Thus, the original results of this study comprise: the design of the adaptive controller and the boundedness and convergence features, shown in Section 4; the simulations, in Section 5; the conclusions in Section 6; the determination and proof of the boundedness features of the control gain, in Section 2; and the statement of the control goal in Section 3.

\section{Background and Model Description}

\subsection{Background: Operation and Control of Fed Batch Processes}

Common goals of fed-batch operation are: (i) To tackle the effect of substrate inhibition and catabolite repression; (ii) to achieve high biomass growth; (iii) to achieve high rate of product formation $[11,31]$. The biomass growth, product formation, and growth rate are highly influenced by the feeding rate. Underfeeding may cause low productivity and starvation, whereas overfeeding may lead to formation of undesired products [11,32,33]. A high feed rate gives faster growth and lower process time, improving productivity, but excess carbon source usually leads to accumulation of metabolic by-products that inhibit cell growth and formation of the target product $[7,10]$. A no feeding phase can be used before the feeding phase, featuring substrate consumption and biomass growth, being the feeding phase initiated when the substrate concentration has decreased until a critical value $[4,34]$. Thus, the aforementioned goals can be achieved by either open loop operation, optimization or control of biochemical variables, with adequate manipulation of the feeding rate [11,32]. To this end, development and utilization of an appropriate kinetic description of involved bioprocess phenomena is required. The cell population can be described through stochastic models involving a probabilistic function that consider the probability of birth and death of microbial cells depending on the cell age. If probabilistic functions are not considered in the model, the description becomes deterministic and other types of distribution can be used, such as the distribution of the average age of cell population as a function of time. The kinetic models of the culture behavior can be either structured or nonstructured. In the former case, the constituent components of the biological system studied and the interactions between them are considered. These models are complex and the determination of their parameters is quite difficult. In contrast, the nonstructured models simplify the description of culture behavior by considering the cells as black boxes with some "average" composition that is characterized through the overall cell biomass concentrations [35,36]. However, optimization and control are hampered by modelling mismatch, and control is also hampered by feed disturbances and measurement noise. Modeling mismatch is caused by complex biochemical processes occurring during microbial growth and synthesis of metabolites, being highly sensitive to culture conditions. As a consequence, the development of reliable mechanistic models is challenging, with changing observed kinetics, unknown parameter variation, and commonly poor model predictions $[7,9,17,37]$. In addition, in bioreactor controllers that include integral action, input saturation may lead to degraded performance of the closed loop system [38,39].

Conventionally, the control of biochemical variables in fed-batch processes is open loop type (feed-forward), using an exponential feeding rate for regulation of the specific growth rate, the set point value being chosen so as to avoid formation of undesired product. 
Nevertheless, this approach is robust neither to process disturbances nor to modelling error $[10,40]$. In on-line optimization a nonlinear programming problem through control vector parameterization (CVP) approach is often considered [41,42]. In closed loop control, on-line feedback of the tracking error is used, with the feed rate as the manipulated variable, and either substrate concentration, product concentration, or specific growth rate as controlled variable $[10,40]$. One possibility is to regulate substrate concentration so as to favour either biomass growth or product formation. In this case, the set point value must be chosen according to the objective of the process [10]. One way to avoid formation of undesired product is to on-line measure its concentration, and to reduce the feed rate if threshold values are exceeded [10]. In particular, adaptive control of bioreactors uses online parameter adaptation, in order to overcome the lack of knowledge on kinetics [11,38]. Model reference adaptive control (MRAC) methods allow handling model non-linearities, unpredictable system dynamics, and unpredictable disturbances. The control design is aimed at minimizing the error between the desired and measured output (tracking error). The resulting control and update mechanisms depend on the tracking error [11]. The approach of [14] uses the MRAC scheme as framework, and a Lyapunov function with dead-zone quadratic forms, in order to tackle the lack of knowledge on varying model parameters, including unknown varying control gain.

\subsection{Model Description}

We consider the fed-batch model [43], which corresponds to the model of $[6,10,44,45]$ with zero maintenance constant, that is, $m_{s}=0$ :

$$
\begin{gathered}
\frac{d S}{d t}=-(Y \mu) X+\left(S_{i n}-S\right) \frac{Q_{i n}}{V} \\
\frac{d X}{d t}=\mu X+(-1) X \frac{Q_{i n}}{V} \\
\frac{d V}{d t}=Q_{i n}
\end{gathered}
$$

where $S$ is the substrate concentration, $X$ is the biomass concentration, $\mu$ is the specific growth rate of biomass, $Y$ is the yield coefficient, $S_{i n}$ is the inflow substrate concentration, $Q_{\text {in }}$ is the inlet flowrate, $V$ is the volume of the culture medium. Common expressions of specific reaction rates used for biological systems include Contois, Monod, and Haldane [10,46-48]. Specific reaction rates can be negative for $X>0$, see [49-51].

The inlet flowrate $Q_{i n}$ is chosen as control input. In practical bioreactor operation, it is non-negative and must have an upper bound defined by the operational limits of the pumps [52]. Therefore, the relationship between $Q_{i n}$ (denoted as $v$ ) and the designed unconstrained control input (denoted as $u$ ) is:

$$
v=\left\{\begin{array}{l}
u_{\max } \text { if } u>u_{\max } \\
u \text { if } u \in\left[u_{\min }, u_{\max }\right] \\
u_{\min } \text { if } u<u_{\min }
\end{array}\right.
$$

Remark 1. In the case of no saturation, we have $v=u$, so that $\Delta u=v-u$ is zero. In the case of saturation at the upper bound, $v=u_{\max }$, we have: $z<-C_{b e}, u>u_{\max }$. Therefore, $\Delta u=(-1)\left(u-u_{\max }\right)<0$. In the case of saturation at the lower bound, $v=u_{\min }$, we have: $z>C_{\text {be }}, u<u_{\text {min }}$. Therefore, $\Delta u=u_{\text {min }}-u>0$.

The following features are considered:

Assumption 1. The substrate concentration $S$ and the biomass concentration $X$ are bounded, $X \in \mathbb{R}^{+}$and $S \in\left(0, S_{\text {in }}\right)[6]$.

Assumption 2. The value of $Y$ is positive and unknown, and its upper bounds are also unknown $[3,15,45]$. 
Assumption 3. The specific growth rate $\mu$ is a generic function, it is unknown, bounded, and its upper and lower bounds are unknown $[16,53,54]$.

Assumption 4. The biomass concentration $X$ is unknown, and its upper bound is also unknown $[3,15,53,55]$.

Assumption 5. The value of $Q_{i n}$ is measured in real time without noise, whereas the values of $S_{i n}$, $S, V$ are noisily measured in real time, and the measurement noise models are:

$$
\begin{gathered}
S_{i m}=S_{i n}+\delta_{s i} \\
S_{m}=S+a_{0} \\
V_{m}=V+\delta_{v}
\end{gathered}
$$

where $S_{i n}, S, V$ are unknown real values, whereas $S_{i m}, S_{m}, V_{m}$ are the noisy measurements, and $\delta_{s i}$, $a_{0}, \delta_{v}$ are the measurement errors, respectively. The measurement errors and their time derivatives are unknown, bounded, and their bounds are unknown. The measured values $S_{i m}, S_{m}$ satisfy $\left(S_{i m}-S_{m}\right)>0$ according to $[53,54,56]$. The measured value of the volume satisfies $V_{m}>0$.

The measurement models (5) to (7) were stated based on the measurement model structure $(\cdot)_{m}=(\cdot)+\delta$, where $(\cdot)_{m}$ is the measured value, $(\cdot)$ is the real value, and $\delta$ is the measurement error [57].

Remark 2. The conditions considered in Assumption 1 are accomplished for fed-batch reactor with control of substrate concentration:

- Real control applications. The substrate and biomass concentrations remain positive and bounded, and the inflow substrate concentration is lower than the substrate concentration in real control applications (see [37]).

- Model-based applications. For model (1) to (3), the state variables X, S are upper bounded for bounded dilution rate $Q_{i n} / V(c f[16,38])$, and $X \geq 0, S<S_{\text {in }}[6]$. Moreover, substrate concentration is kept positive when substrate concentration is controlled [10]. During fedbatch regime only partial equilibria are possible: biomass or substrate concentrations can be stabilized, but $V$ cannot, because $Q_{\text {in }} \geq 0$ so that $V>0$ [16].

Remark 3. Assumptions 2 and 3 are related to the uncertainty on the reaction rate terms, which is due to the unknown time varying nature of the reaction yield and coefficients of the reaction rates [3-6]. In case of fed-batch reactors, it has been reported that unknown parameter variation arises as a consequence of complex biochemical processes that occur during microbial growth and synthesis of metabolites, being highly sensitive to culture conditions $[7,9,17,37]$.

Remark 4. Assumption 5 is related to measurement noise. On-line measurement of biological state variables, e.g., concentrations of substrates, biomass, metabolic enzymes and product is severely affected by the following: the required quality of monitored data and sampling frequency depend on the accuracy of the sensors employed; in many cases, online measurement of the state variables is not possible, due to the high cost of sensors and extreme operating conditions [58]. Measurement noise can be expressed in additive form [16].

Remark 5. The online measurement of substrate concentration and the unavailability of biomass concentration (in Assumptions 4 and 5) are realistic conditions in control of substrate concentration [15]. 


\subsection{Features of the Control Gain}

In this subsection, we prove some features of the control gain that are required by the controller design. Since $S_{i n}, V$ measurements are noisy, the control gain $b=\left(S_{i n}-S\right) / V$ is computed as:

$$
b_{m}=\frac{S_{i m}-S_{m}}{V_{m}}
$$

In order to account for this, we rewrite $b$ in terms of $b_{m}$, using the measurement models (5) to (7):

$$
b=b_{\delta} b_{m}
$$

where

$$
b_{\delta}=\frac{S_{i}-S}{S_{i m}-S_{m}} \frac{V_{m}}{V}
$$

$b_{\delta}$ is unknown, whereas $b_{m}$ is known. The following properties hold:

$$
\begin{gathered}
P i) b_{\delta}>\mu_{b}>0 \\
\mu_{b}=\frac{\min \left\{V_{m}\right\}}{\min \left\{V_{m}\right\}+\max \left|\delta_{v}\right|} \cdot \frac{\min \left\{S_{i}-S\right\}}{\max \left\{S_{i}\right\}+\max \{|\delta|\}}>0 \\
\text { Pii) } b_{m}>\frac{\min \left\{S_{i m}-S_{m}\right\}}{\max \left\{V_{m}\right\}}>0
\end{gathered}
$$

Piii) $b_{\delta}$ is upper bounded

where $\mu_{b}$ is positive, constant, and unknown. The proof is provided at what follows. From (7) it follows that $V<V_{m}+\max \left|\delta_{v}\right|$. Hence

$$
\begin{gathered}
\frac{V_{m}}{V}>\frac{V_{m}}{V_{m}+\max \left|\delta_{v}\right|} \\
\frac{V_{m}}{V}>\frac{\min \left\{V_{m}\right\}}{\min \left\{V_{m}\right\}+\max \left|\delta_{v}\right|}>0
\end{gathered}
$$

Since $S_{i m}-S_{m}<S_{i m}$, using (5), we get:

$$
S_{i m}-S_{m}<\max \left\{S_{i}\right\}+\max |\delta|
$$

Assumption 1 implies $S_{i}-S>0$. Using this fact, Equation (16) and Assumption 5, we get:

$$
\begin{gathered}
\frac{1}{S_{i m}-S_{m}}>\frac{1}{\max \left\{S_{i}\right\}+\max \{|\delta|\}} \\
\frac{S_{i}-S}{S_{i m}-S_{m}}>\frac{\min \left\{S_{i}-S\right\}}{\max \left\{S_{i}\right\}+\max \{|\delta|\}}>0
\end{gathered}
$$

Combining Equations (15) and (17), yields Equation (11).

To prove that $b_{m}$ is bounded away from zero, we notice that the bounded nature of $V_{m}$ leads to

$$
\frac{1}{V_{m}}>\frac{1}{\max \left\{V_{m}\right\}}
$$

which implies that Equation (13) holds.

To conclude the bounded nature of $\delta_{b}(10)$ stated in property (14), we notice from Assumptions 1 and 5. that: $\delta_{s i}, a_{0}, \delta_{v}$ are bounded; $\left(S_{i m}-S_{m}\right)>0 ; V>0$ and $S$ is bounded for $Q_{i n}$ bounded. 


\section{Reference Model and Control Goal}

\subsection{Reference Model}

In adaptive control of bioreactors, the beginning of closed loop operation exhibits a higher increase of updated parameters and a higher control effort, which in turn implies a higher possibility of input saturation $[14,15,54]$. To reduce this effect, one strategy is to set up the desired behavior of the output, denoted as $y_{d}$, with continuous evolution from the initial value $\left.y_{d}\right|_{t_{0}}=\left.y\right|_{\text {to }}$ to the expected set point, being $t_{0}$ the time the controller is started $($ see $[6,14])$. To that end, we use the reference model $y_{d}=\left(a_{m} /\left(p+a_{m}\right)\right) \bar{r}$, equivalently,

$$
\frac{d y_{d}}{d t}=a_{m}\left(-y_{d}+\bar{r}\right)
$$

where $\bar{r}$ is the command signal and $a_{m}$ is a positive constant defined by the user, which determines the speed in which the desired output $y_{d}$ approaches the signal $\bar{r}$. We choose the following conditions on $\bar{r}$ and $y_{d}$ :

$$
\left.\bar{r}\right|_{t o}=\left.y\right|_{t o} ;\left.y_{d}\right|_{t o}=\left.\bar{r}\right|_{t o}
$$

Remark 6. The desired output $y_{d}$ is provided by model (18), with conditions (19), so that $y_{d}, \dot{y}_{d}$ are bounded and known.

Remark 7. From (19) it follows that $\left.e\right|_{\text {to }}=0$ which implies lower control effort at initial time.

\subsection{Control Goal}

Control goal. Consider: (i) the plant model (1)-(3) subject to Assumptions 1 to 5, being $S$ the controlled output $(y=S)$ and $Q_{i n}$ the control input, so that

$$
y_{m}=y+a_{o}
$$

$y_{m}$ is the noisy measurement of $y$ and $y$ is the unknown real output value, according to Assumption 5.

(ii) the tracking error $e$

$$
e=y_{m}-y_{d}
$$

where $y_{d}$ is the desired output, provided by model (18), satisfying conditions (19), with positive constant user-defined $a_{m}$ and user-defined $\bar{r}$; (iii) the residual set $\Omega_{e}=\left\{e:|e| \leq C_{b e}\right\}$ whose size $C_{b e}$ is positive, constant, and user-defined.

The goal of the controller design is to formulate a control law and an update law for the control input $v$ which corresponds to $Q_{i n}$, such that: (Gi) the tracking error $e$ converges asymptotically to the residual set $\Omega_{e}$; (Gii) the updated parameter, the tracking error and control law are bounded upon closed loop (parameter drifting is avoided); (Giii) the control and update laws involve no discontinuous signals.

Remark 8. The reason for stating condition Giii is that the presence of discontinuous signals in the control input may lead to input chattering, problems of existence and uniqueness of closed loop trajectories, and the need of using Filippov theory, as discussed by $[59,60]$.

\section{Controller Design and Stability Analysis}

In this section, the adaptive robust controller is designed for the bioreactor model (1)-(3) subject to Assumptions 1 to 5, the control goal stated in Section 3, and the input constraint (4). Some important tasks of the procedure are: (i) dead-zone quadratic forms are used instead of the current Lyapunov function; (ii) the unknown varying model parameters are parameterized in terms of the control gain, in order to tackle the unknown varying nature of the control gain; (iii) a new auxiliary system is proposed in order to tackle both the effect of input saturation and uncertainty on the control gain; (iv) a new treatment of the $b \Delta u$ 
term is proposed; $(\mathrm{v})$ a saturation function of the modified tracking error is used instead of discontinuous functions.

For simplicity seek, the substrate Equation (1) is combined with definition (9):

$$
\frac{d S}{d t}=-(Y \mu) X+b_{\delta} b_{m} v
$$

where $b_{m}$ is known, whereas $b_{\delta}$ is unknown, and both are bounded away from zero, according to properties (11) to (14). Consider the modified error $z=e-\psi$, where $\psi$ is the state of the auxiliary system that will be defined later. Accounting for the tracking error $e=y_{m}-y_{d}$ yields

$$
z=y_{m}-y_{d}-\psi
$$

Combining with Equation (20) yields $z=y+a_{o}-y_{d}-\psi$. Differentiating with respect to time, yields $\dot{z}=\dot{y}-\dot{y}_{d}-\dot{\psi}+\dot{a}_{o}$. Combining this with Equation (22) yields

$$
\frac{d z}{d t}=-(Y \mu) X+\dot{a}_{0}-\dot{y}_{d}+b_{\delta} b_{m} v-\dot{\psi}
$$

To handle the uncertainty on $Y, \mu, X, a_{0}, \dot{a}_{0}$, the following Lyaunov-like function is used:

$$
V_{z}=\left\{\begin{array}{l}
(1 / 2)\left(z-C_{b e}\right)^{2} \text { for } z \geq C_{b e} \\
0 \text { for } z \in\left(-C_{b e}, C_{b e}\right) \\
(1 / 2)\left(z+C_{b e}\right)^{2} \text { for } z \leq-C_{b e}
\end{array}\right.
$$

which vanishes for $z \in\left(-C_{b e}, C_{b e}\right)$. An early form of this function is reported by $[61,62]$, and latter variants are reported by [14,63-65]. The main properties of $V_{z}$ are:

$$
\begin{gathered}
V_{z} \geq 0 \\
|z| \leq C_{b e}+\sqrt{2 V_{z}}
\end{gathered}
$$

$V_{z}, d V_{z} / d e$ are continuous with respect to $z$

Differentiating (25) with respect to time, yields

$$
\begin{gathered}
\frac{d V_{z}}{d t}=f_{z} \frac{d z}{d t} \\
f_{z}=\frac{d V_{z}}{d z}=\left\{\begin{array}{c}
z-C_{b e} \text { for } z \geq C_{b e} \\
0 \text { for } z \in\left(-C_{b e}, C_{b e}\right) \\
z+C_{b e} \text { for } z \leq-C_{b e}
\end{array}\right.
\end{gathered}
$$

Combining Equations (29) and (24) and adding and subtracting the term $c_{1} f_{z}^{2}$, yields

$$
\frac{d V_{z}}{d t}=f_{z} \dot{z}=-c_{1} f_{z}^{2}+\left(-(Y \mu) X+\dot{a}_{o}\right) f_{z}+\left(c_{1} f_{z}-\dot{y}_{d}\right) f_{z}+b_{\delta} b_{m} v f_{z}-f_{z} \dot{\psi}
$$

being $c_{1}$ a user-defined positive constant. The term $-c_{1} f_{z}^{2}$ provides asymptotic convergence of $f_{z}^{2}$ to zero, as is shown later in the convergence analysis.

The input $v(4)$ is expressed as $v=u+\Delta u$, where:

$$
\Delta u=v-u
$$

Therefore, $b_{\delta} b_{m} v f_{z}=b_{\delta} b_{m} u f_{z}+b_{\delta} b_{m} \Delta u f_{z}$. Substituting this into Equation (31), yields

$$
\frac{d V_{z}}{d t}=-c_{1} f_{z}^{2}+\left(-(Y \mu) X+\dot{a}_{o}\right) f_{z}+\left(c_{1} f_{z}-\dot{y}_{d}\right) f_{z}+b_{\delta} b_{m} u f_{z}+b_{\delta} b_{m} \Delta u f_{z}-f_{z} \dot{\psi}
$$


The term $b_{\delta}$ is upper bounded according to property (14), so that it satisfies

$$
\left|b_{\delta}\right| \leq \bar{\mu}_{2}
$$

where $\bar{\mu}_{2}$ is unknown, positive, and constant. Therefore,

$$
b_{\delta} b_{m} \Delta u f_{z} \leq \bar{\mu}_{2}\left|b_{m} f_{z} \Delta u\right|
$$

In order to tackle the lack of knowledge on $\bar{\mu}_{2}$, we define the updating error

$$
\widetilde{\theta}_{b}=\hat{\theta}_{b}-\bar{\mu}_{2}
$$

where $\hat{\theta}_{b}$ is an updated parameter that will be defined later; it allows tackling the lack of knowledge on $b_{\delta}$. From the above expression, $\bar{\mu}_{2}$ can be expressed as $\bar{\mu}_{2}=\hat{\theta}_{b}-\widetilde{\theta}_{b}$. Substituting this into (35), yields

$$
b_{\delta} b_{m} \Delta u f_{z} \leq\left|b_{m} f_{z} \Delta u\right| \hat{\theta}_{b}-\left|b_{m} f_{z} \Delta u\right| \widetilde{\theta}_{b}
$$

Substituting into Equation (33) and arranging, yields

$$
\begin{gathered}
\frac{d V_{z}}{d t} \leq-c_{1} f_{z}^{2}+\left(-(Y \mu) X+\dot{a}_{o}\right) f_{z}+\left(c_{1} f_{z}-\dot{y}_{d}\right) f_{z}+b_{\delta} b_{m} u f_{z} \\
-f_{z}\left(\dot{\psi}-\operatorname{sgn}\left(f_{z}\right)\left|b_{m} \Delta u\right| \hat{\theta}_{b}\right)-\left|b_{m} f_{z} \Delta u\right| \widetilde{\theta}_{b}
\end{gathered}
$$

Therein, the signal $\operatorname{sgn}\left(f_{z}\right)$ is discontinuous with respect to $z$, as can be concluded from (30). This would lead to a discontinuous signal in the definition of $\dot{\psi}$. To remedy this, we notice from (30) that $\left|f_{z}\right|$ can be rewritten as:

$$
\left|f_{z}\right|=f_{z} s a t_{z}
$$

where

$$
\text { sat }_{z}=\left\{\begin{array}{l}
+1 \text { for } z \geq C_{b e} \\
z / C_{b e} \text { for } z \in\left(-C_{b e}, C_{b e}\right) \\
-1 \text { for } z \leq-C_{b e}
\end{array}\right.
$$

Using this property, Equation (38) can be rewritten as:

$$
\begin{gathered}
\frac{d V_{z}}{d t} \leq-c_{1} f_{z}^{2}+\left(-(Y \mu) X+\dot{a}_{o}\right) f_{z}+\left(c_{1} f_{z}-\dot{y}_{d}\right) f_{z}+b_{\delta} b_{m} u f_{z} \\
-f_{z}\left(\dot{\psi}-s a t_{z}\left|b_{m} \Delta u\right| \hat{\theta}_{b}\right)-\left|b_{m} f_{z} \Delta u\right| \widetilde{\theta}_{b}
\end{gathered}
$$

Thus, in order to counteract the effect of the term involving $\left|b_{m} \Delta u\right| \hat{\theta}_{b}$, the auxiliary system is chosen as

$$
\frac{d \psi}{d t}=-c_{2} \psi+s a t_{z} \hat{\theta}_{b}\left|b_{m} \Delta u\right|
$$

where $c_{2}$ is a user-defined positive constant, $\Delta u$ is defined in Equation (32) and $\hat{\theta}_{b}$ is an updated parameter that is defined later, and it allows tackling the lack of knowledge on $b_{\delta}$.

Remark 9. The main differences of this auxiliary system with respect to that of [24] are: (i) it involves the saturation function sat $z_{z}$, (ii) it involves the $b_{m}$ term instead of considering the control gain as completely unknown; (iii) it involves an absolute value term that includes the $\Delta u$ signal. This features are due to the robustness strategy.

Substituting Equation (42) into Equation (41) and arranging, yields

$$
\frac{d V_{z}}{d t} \leq-c_{1} f_{z}^{2}+\left(-(Y \mu) X+\dot{a}_{o}\right) f_{z}+\left(c_{1} f_{z}-\dot{y}_{d}+c_{2} \psi\right) f_{z}+b_{\delta} b_{m} u f_{z}-\widetilde{\theta}_{b}\left|b_{m} f_{z} \Delta u\right|
$$


The application of the robustness strategy of [14] would imply $\operatorname{sgn}(u)=(-1) \operatorname{sgn}\left(b_{\delta}\right)$ $\operatorname{sgn}(z)$ for $z \neq 0$. Definition (10) and constraint (4) lead to $\operatorname{sgn}\left(b_{\delta}\right)=1$ and $\min (v)=0$, so that $\operatorname{sgn}(u)=-\operatorname{sgn}(z)$. Therefore, $u<0$ and $v=0$ whenever $z>0$. This would imply frequent saturation in the long term, corresponding to moments when $z>0$. In order to reduce this effect, an additive constant term $u_{a}$ is incorporated in the control input:

$$
b_{m} u=u_{a}+u_{b}
$$

where $u_{a}$ is constant, positive, user-defined, and satisfies $u_{a} \in\left[u_{\min }, u_{\max }\right]$, and its value can be selected as the value of $u$ at initial open loop regime. Equation (44) leads to

$$
u=\frac{1}{b_{m}}\left(u_{a}+u_{b}\right)
$$

Substituting into Equation (43), yields

$$
\frac{d V_{z}}{d t} \leq-c_{1} f_{z}^{2}+\left(-(Y \mu) X+\dot{a}_{o}+b_{\delta} u_{a}\right) f_{z}+\left(c_{1} f_{z}-\dot{y}_{d}+c_{2} \psi\right) f_{z}+b_{\delta} u_{b} f_{z}-\widetilde{\theta}_{b}\left|b_{m} f_{z} \Delta u\right|
$$

In view of Assumptions 1 to 5 , the term $-(Y \mu) X+\dot{a}_{0}+b_{\delta} u_{a}$ is bounded, so that $-(Y \mu) X+\dot{a}_{o}+b_{\delta} u_{a} \leq \bar{\mu}_{1}$, where its upper bound $\bar{\mu}_{1}$ is unknown, positive, and constant. Therefore,

$$
\begin{gathered}
\left(-(Y \mu) X+\dot{a}_{o}+b_{\delta} u_{a}\right) f_{z} \leq\left|f_{z}\right| \bar{\mu}_{1} \\
\left(c_{1} f_{z}-\dot{y}_{d}+c_{2} \psi\right) f_{z} \leq\left|c_{1} f_{z}-\dot{y}_{d}+c_{2} \psi\right|\left|f_{z}\right|
\end{gathered}
$$

In order to account for the presence of the unknown $b_{\delta}$, we incorporate the lower bound $\mu_{b}$, defined in Equation (12):

$$
\begin{gathered}
\left(-(Y \mu) X+\dot{a}_{o}+b_{\delta} u_{a}\right) f_{z} \leq \mu_{b}\left|f_{z}\right| \frac{\bar{\mu}_{1}}{\mu_{b}} \\
\left(c_{1} f_{z}-\dot{y}_{d}+c_{2} \psi\right) f_{z} \leq \mu_{b}\left|f_{z}\right|\left|c_{1} f_{z}-\dot{y}_{d}+c_{2} \psi\right| \frac{1}{\mu_{b}}
\end{gathered}
$$

where $\bar{\mu}_{1} / \mu_{b},\left(1 / \mu_{b}\right)$ are unknown, positive, and constant. Adding Equations (48) and (49), yields

$$
\left(-(Y \mu) X+\dot{a}_{o}+b_{\delta} u_{a}\right) f_{z}+\left(c_{1} f_{z}-\dot{y}_{d}+c_{2} \psi\right) f_{z} \leq \mu_{b}\left|f_{z}\right|\left(\frac{\bar{\mu}_{1}}{\mu_{b}}+\left|c_{1} f_{z}-\dot{y}_{d}+c_{2} \psi\right| \frac{1}{\mu_{b}}\right)=\mu_{b} \varphi^{\top} \theta\left|f_{z}\right|
$$

where

$$
\begin{gathered}
\varphi=\left[1,\left|c_{1} f_{z}-\dot{y}_{d}+c_{2} \psi\right|\right]^{\top} \\
\theta=\left[\frac{\bar{\mu}_{1}}{\mu_{b}}, \frac{1}{\mu_{b}}\right]^{\top}
\end{gathered}
$$

Remark 10. To tackle the lack of knowledge on the input gain term $b_{\delta}$, a crucial task was to incorporate the lower bound $\mu_{b}$ in the expression of uncertain model parameters in terms of upper bounds [Equations (48) and (49)], and in the definition of $\theta$ [Equations (50) and (52)].

In order to rewritte $\varphi^{\top} \theta$ in terms of updating errors and updated parameters, we define the updating error as:

$$
\widetilde{\theta}=\hat{\theta}-\theta
$$

where $\hat{\theta}$ is an updated parameter that will be defined later.

Remark 11. The size of the $\hat{\theta}$ vector is the same as that of $\theta$, and it is not increased by the incorporation of the additive term $u_{a}$ in the control input. 
The parameter vector $\theta$ can be expressed as $\theta=\hat{\theta}-\widetilde{\theta}$, and $\varphi^{\top} \theta$ can be expressed as $\varphi^{\top} \theta=-\tilde{\theta}^{\top} \varphi+\varphi^{\top} \hat{\theta}$. Substituting this into Equation (50), yields:

$$
\left(-(Y \mu) X+\dot{a}_{o}+b_{\delta} u_{a}\right) f_{z}+\left(c_{1} f_{z}-\dot{y}_{d}+c_{2} \psi\right) f_{z} \leq-\mu_{b}\left|f_{z}\right| \widetilde{\theta}^{\top} \varphi+\mu_{b}\left|f_{z}\right| \varphi^{\top} \hat{\theta}
$$

In view of Property (11), we have $\mu_{b}\left|f_{z}\right| \varphi^{\top} \hat{\theta} \leq\left|b_{\delta}\right|\left|f_{z}\right|\left|\varphi^{\top} \hat{\theta}\right|$. Substituting this into Equation (54), yields

$$
\left(-(Y \mu) X+\dot{a}_{o}+b_{\delta} u_{a}\right) f_{z}+\left(c_{1} f_{z}-\dot{y}_{d}+c_{2} \psi\right) f_{z} \leq-\mu_{b}\left|f_{z}\right| \widetilde{\theta}^{\top} \varphi+\left|b_{\delta}\right|\left|f_{z}\right|\left|\varphi^{\top} \hat{\theta}\right|
$$

Substituting into Equation (46) and arranging, yields

$$
\frac{d V_{z}}{d t} \leq-c_{1} f_{z}^{2}-\mu_{b}\left|f_{z}\right| \widetilde{\theta}^{\top} \varphi+b_{\delta} f_{z}\left(u_{b}+\operatorname{sgn}\left(b_{\delta}\right) \operatorname{sgn}\left(f_{z}\right)\left|\varphi^{\top} \hat{\theta}\right|\right)+(-1) \widetilde{\theta}_{b}\left|b_{m} f_{z} \Delta u\right|
$$

Thus, the control law term can be defined as $u_{b}=-\operatorname{sgn}\left(b_{\delta}\right) \operatorname{sgn}\left(f_{z}\right)\left|\varphi^{\top} \hat{\theta}\right|$, so as to obtain $d V_{z} / d t \leq-c_{1} f_{z}^{2}-\mu_{b}\left|f_{z}\right| \widetilde{\theta}^{\top} \varphi+(-1) \widetilde{\theta}_{b} \mid f_{z} b_{m} \Delta u$. Since it involves the discontinuous signal $\operatorname{sgn}\left(f_{z}\right)$, we use the property (39), so that (55) can be rewritten as:

$$
\frac{d V_{z}}{d t} \leq-c_{1} f_{z}^{2}-\mu_{b}\left|f_{z}\right| \widetilde{\theta}^{T} \varphi+b_{\delta} f_{z}\left(u_{b}+\operatorname{sgn}\left(b_{\delta}\right) s a t_{z}\left|\varphi^{\top} \hat{\theta}\right|\right)+(-1) \widetilde{\theta}_{b}\left|b_{m} \Delta u f_{z}\right|
$$

Thus, the control law term $u_{b}$ is chosen as:

$$
u_{b}=-\operatorname{sgn}\left(b_{\delta}\right) \operatorname{sat}_{z}\left|\varphi^{\top} \hat{\theta}\right|
$$

Using (45), the control law is:

$$
\begin{aligned}
& u=\frac{1}{b_{m}}\left(u_{a}-\operatorname{sgn}\left(b_{\delta}\right) \operatorname{sat}_{z}\left|\varphi^{\top} \hat{\theta}\right|\right) \\
& v=\left\{\begin{array}{l}
u_{\text {max }} \text { if } u>u_{\text {max }} \\
u \text { if } u \in\left[u_{\text {min }}, u_{\text {max }}\right] \\
u_{\text {min }} \text { if } u<u_{\text {min }}
\end{array}\right.
\end{aligned}
$$

which is continuous with respect to $z$ and $\hat{\theta}$. Substituting $u_{b}$ (57) into Equation (56), yields

$$
\frac{d V_{z}}{d t} \leq-c_{1} f_{z}^{2}-\mu_{b}\left|f_{z}\right| \widetilde{\theta}^{\top} \varphi+(-1) \widetilde{\theta}_{b}\left|b_{m} \Delta u f_{z}\right|
$$

The update law is chosen so as to tackle the effect of the term $-\mu_{b}\left|f_{z}\right| \widetilde{\theta}^{\top} \varphi$ :

$$
\dot{\hat{\theta}}=\Gamma \varphi\left|f_{z}\right|
$$

where $\Gamma$ is a $2 \times 2$ diagonal matrix whose diagonal entries are user defined positive constants. The update law for $\hat{\theta}_{b}$ is chosen so as to tackle the effect of the term $(-1) \widetilde{\theta}_{b}\left|b_{m} \Delta u f_{z}\right|$ :

$$
\dot{\hat{\theta}}_{b}=\gamma_{b}\left|b_{m} f_{z} \Delta u\right|
$$

where $\gamma_{b}$ is user defined, positive, and constant.

Remark 12. The controller comprises: the control law (4), (58); the update law (60) with the $2 \times 2$ diagonal matrix $\Gamma$ whose diagonal entries are user defined, positive, and constant; the update law (61) being $\gamma_{b}$ user defined, positive, and constant; and the auxiliary system (42). The signals involved therein are: (i) $e$ (21), $z(23), f_{z}(30), \varphi(51)$, sat $z$ (40), $b_{m}$ (8); (ii) the desired output $y_{d}$, provided by model (18), with conditions (19), according to remark 6.; (iii) the input error $\Delta u$ (32), 
which involves $u$ (58) and $v(4)$; (iv) the constants $c_{1}$ and $c_{2}$, which are user-defined and positive; (v) the constant $u_{a}$, which is user-defined, positive, and satisfies $u_{a} \in\left[u_{\text {min }}, u_{\text {max }}\right]$.

Remark 13. In the control design procedure, a new treatment of the $b \Delta u f_{z}$ term is proposed, in view of the lack of knowledge on $b$ and its upper bound $\bar{\mu}_{2}$ : (i) the $b \Delta u f_{z}$ term is expressed as function of its upper bound [Equation (35)]; (ii) the $b \Delta u f_{z}$ term is expressed as function of parameter updating error $\widetilde{\theta}_{b}$ and updated parameter $\hat{\theta}_{b}$ [Equation (37)]; (iii) the update law for $\hat{\theta}_{b}$ [Equation (61)] is formulated so as to obtain adequate time derivative of the overall Lyapunov function.

Remark 14. The resulting auxiliary system (42) involves significant differences with respect to current ones $([25,29])$, and also different with respect to that of [30]: it involves a saturation function of $z$; it involves the updated parameter $\hat{\theta}_{b}$, which is function of $f_{z}$ and $\Delta u$; the $\Delta u$ signal is in absolute value.

Remark 15. Other major features of the control design procedure are:

- $\quad$ The modified error $z=e-\psi$ is used as closed loop state variable instead of the tracking error $e$, being $\psi$ provided by the auxiliary system (42);

- In the $\dot{V}_{z}$ expression, the effect of $b \Delta u f_{z}$ term is tackled by means of the term $-f_{z} \dot{\psi}$ by properly defining the auxiliary system;

- Due to the lack of knowledge on the lower bound of $b_{\delta}$, that is $\mu_{\delta}, \mu_{\delta}$ is incorporated in the arrangement of uncertain model terms as function of upper bounds [Equations (48) and (49)]; and $\mu_{\delta}$ is incorporated in the definition of the unknown parameter vector [Equation (52)];

- Due to the the lack of knowledge on the upper bounds of uncertain model terms, the model terms are arranged as function of upper bounds [Equation (47)]; the model terms are arranged as function of parameter and regression vector [Equation (50) to (52)]; the model terms are arranged as function of parameter updating error and updated parameters [Equation (54)]; and the update law for $\hat{\theta}$ [Equation (60)] is defined.

Remark 16. The main features of the formulated control law, update law, and auxiliary system are: (i) The structure of the reaction rate terms, the plant model coefficients, the control gain and the upper and lower bounds of model terms are not required to be known; (ii) the known term $b_{m}$ (8) is used instead of considering the control gain b as completely unknown; (iii) instead of using discontinuous signals, a saturation function of the modified error $z$ is used in the control law and the auxiliary system, and the continuous function $f_{z}$ is used in the update laws, in this way undesired input chattering and the need of using the Filippov theory are avoided.

Remark 17. When $z$ is inside the target region $\Omega_{z}=\left\{z:|z| \leq C_{b e}\right\}, f_{z}=0$, so that $\hat{\theta}$ remains constant, which is concluded from Equations (60) and (30). In turn, this contributes to the avoidance of parameter drifting.

\subsection{Control Goal}

Theorem 1. [Boundedness of the closed loop signals]. Consider the model (1)-(3), subject input constraint (4) and Assumptions 1 to 5, and signals e (21), $z$ (23), $f_{z}(30), \varphi(51)$, sat $z$ (40). If the controller (58), (60), (61), (42) is applied, then: (Ti) the signals $z, \hat{\theta}, \hat{\theta}_{b}, e, \psi$ and $b_{m} u$ remain bounded; (Tii) $|z| \leq C_{b e}+\sqrt{2 W\left(\bar{x}_{t o}\right)}$; (Tiii) $\left|\hat{\theta}_{b}\right| \leq \sqrt{2 \gamma_{b} W\left(\bar{x}_{t o}\right)}+\bar{\mu}_{2}$.

Proof of Theorem 1. The Lyapunov function is chosen as

$$
\begin{gathered}
W(\bar{x})=V_{z}+V_{\theta} \\
V_{\theta}=\frac{1}{2} \mu_{b} \widetilde{\theta}^{\top} \Gamma^{-1} \widetilde{\theta}+\frac{1}{2} \gamma_{b}^{-1} \widetilde{\theta}_{b}^{2} \\
\bar{x}=\left[z, \widetilde{\theta}^{\top}\right]
\end{gathered}
$$


where $V_{z}$ is defined in (25), $\widetilde{\theta}$ in (53) and $\widetilde{\theta}_{b}$ in (36). Differentiating $W$ with respect to time, yields

$$
\begin{gathered}
\dot{W}=\dot{V}_{z}+\dot{V}_{\theta} \\
\dot{V}_{\theta}=\frac{1}{2} \mu_{b}\left(\dot{\tilde{\theta}}^{\top} \Gamma^{-1} \widetilde{\theta}+\widetilde{\theta}^{\top} \Gamma^{-1} \dot{\tilde{\theta}}\right)+\gamma_{b}^{-1} \widetilde{\theta}_{b} \dot{\hat{\theta}}_{b}
\end{gathered}
$$

Combining this with update laws (60), (61) yields $\dot{V}_{\theta}=\mu_{b}\left|f_{z}\right| \varphi^{\top} \widetilde{\theta}+\widetilde{\theta}_{b}\left|b_{m} f_{z} \Delta u\right|$. Combining this with Equations (64) and (59), yields

$$
\dot{W} \leq-c_{1} f_{z}^{2}
$$

Arranging and integrating, yields

$$
W(\bar{x}(t))+c_{1} \int_{t_{0}}^{t} f_{z}^{2} d t \leq W\left(\bar{x}\left(t_{0}\right)\right)
$$

Hence, accounting for (26), we have:

$$
\begin{gathered}
W(\bar{x}(t)) \leq W(\bar{x}(t o)) \\
c_{1} \int_{t o}^{t} f_{z}^{2} d t \leq W(\bar{x}(t o))
\end{gathered}
$$

Thus, $W \in L_{\infty}$. In view of (62) one further obtains $V_{z} \in L_{\infty}, V_{\theta} \in L_{\infty}$. In view of (25) one further obtains $z \in L_{\infty}$. In view of (63), (53), (36) one further obtains $\widetilde{\theta} \in L_{\infty}, \widetilde{\theta}_{b} \in L_{\infty}$, and consequently $\hat{\theta} \in L_{\infty}, \hat{\theta}_{b} \in L_{\infty}$.

In view of Assumption $1, X$ and $S$ are bounded since input saturation renders $Q_{i n}$ bounded. Therefore, $y_{m}$ and $e=y_{m}-y_{d}$ are bounded. In view of the definition of $z$ (23), and boundedness of $z$, one further obtains $\psi \in L_{\infty}$. In view of Equation (51), one further obtains $\varphi \in L_{\infty}$. In this way, all the components of $b_{m} u$ are bounded, and using Equation (58) one further obtains $b_{m} u \in L_{\infty}$. This completes the proof of Ti.

We notice that Equations (66) and (62) lead to $V_{z} \leq W\left(\bar{x}_{t o}\right)$. Further using property (27), yields $|z| \leq C_{b e}+\sqrt{2 W\left(\bar{x}_{t o}\right)}$. This completes the proof of Tii.

From Equation (66), (62), (63) it follows that $(1 / 2) \gamma_{b}^{-1} \widetilde{\theta}_{b}^{2} \leq W\left(\bar{x}_{t o}\right)$. Hence,

$$
\left|\widetilde{\theta}_{b}\right| \leq \sqrt{2 \gamma_{b} W\left(\bar{x}_{t o}\right)}
$$

Definition (36) yields $\left|\hat{\theta}_{b}\right| \leq\left|\widetilde{\theta}_{b}\right|+\left|\bar{\mu}_{2}\right|$. Combining this with Equation (68), yields $\left|\hat{\theta}_{b}\right| \leq \sqrt{2 \gamma_{b} W\left(\bar{x}_{t o}\right)}+\bar{\mu}_{2}$. This completes the proof of Tiii.

\subsection{Convergence Analysis}

Theorem 2. [Convergence of signals $z, e$ ]. Consider the model (1)—(3), subject to input constraint (4) and Assumptions 1 to 5, and signals e (21), $z(23), f_{z}(30), \varphi(51)$, sat $z$ (40). If the controller (58), (60), (61), (42) is applied, then: (Ti) the signal $z$ converges asymptotically to $\Omega_{z}$, where $\Omega_{z}=$ $\left\{z:|z| \leq C_{b e}\right\}$; (Tii) if $\Delta u$ vanishes, then e converges asymptotically to $\Omega_{e}, \Omega_{e}=\left\{e:|e| \leq C_{b e}\right\}$.

Proof of Theorem 2. From Equation (67), it follows that $f_{z}^{2} \in L_{1}$. In order to apply the Barbalat's Lemma, we need to prove that $f_{z}^{2} \in L_{\infty}$, and $d\left(f_{z}^{2}\right) / d t \in L_{\infty}$.

Recall that $z \in L_{\infty}$, as stated by Theorem 1. Then, it follows from Equation (30) that $f_{z} \in L_{\infty}$. Hence $f_{z}^{2} \in L_{\infty}$. 
Differentiating $f_{z}^{2}$ with respect to time, using (30), yields:

$$
\frac{d\left(f_{z}^{2}\right)}{d t}=\left\{\begin{array}{c}
2\left(z-C_{b e}\right) \frac{d z}{d t} \text { for } z \geq C_{b e} \\
0 \text { for } z \in\left(-C_{b e}, C_{b e}\right) \\
2\left(z+C_{b e}\right) \frac{d z}{d t} \text { for } z \leq-C_{b e} \\
=2 f_{z} \frac{d z}{d t}
\end{array}\right.
$$

From this we conclude that $d\left(f_{z}^{2}\right) / d t \in L_{\infty}$. So far, we have proved that $\left(f_{z}^{2}\right) \in L_{1}$, $f_{z}^{2} \in L_{\infty}$, and $d\left(f_{z}^{2}\right) / d t \in L_{\infty}$. Applying Barbalat's Lemma [66], yields $\lim _{t \rightarrow \infty}\left(f_{z}^{2}\right)=0$.

Therefore, in view of (30), it follows that $z$ converges asymptotically to $\Omega_{z}$, where $\Omega_{z}=\left\{z:|z| \leq C_{b e}\right\}$. This completes the proof of Ti.

From (42)), it follows that if $\Delta u$ vanishes, then $\psi$ converges to zero. In this case, from Equation (23) it follows that $z$ converges to $e$. In view of the convergence of $z$ stated in $\mathrm{Ti}$, it follows that $e$ converges asymptotically to $\Omega_{e}, \Omega_{e}=\left\{e:|e| \leq C_{b e}\right\}$. This completes the proof of Tii.

The developed controller design considers a fed-batch system, with the substrate concentration as output to be controlled, and the dilution rate as manipulated input. Remarkable features of the system are: (i) the control input is constrained; (ii) the measurements of inflow and outflow substrate concentrations are corrupted by noise, which implies that the control gain is unknown and its bounds are also unknown; (iii) the biomass concentration and the reaction rate parameters are unknown, and their bounds are also unknown. The closed loop features are: (i) The modified tracking error converges to a compact set of user-defined width, which depends on neither model terms, nor modelling error, nor model coefficients nor disturbance terms; (ii) the absence of excessive increase of updated parameters is guaranteed; (iii) discontinuous signals are used in neither the auxiliary system nor the control law nor the update law. To this end, significant improvements were made in the whole control design procedure, and the resulting auxiliary system includes an additional update law, which allows tackling the lack of knowledge on the control gain. In summary, the controller design achieves the aforementioned closed loop features, it takes into account the aforementioned system challenges, and it provides an improved design procedure. This is a significant contribution to current robust control studies for input saturated systems, in special AES-based control designs.

Remark 18. The expression $\dot{W} \leq-c_{1} f_{z}^{2}$ (65) takes into account the formulated controller, that is, the control and update laws, the auxiliary system, and the saturation functions, and the fact that it depends on neither the bounds of external disturbances, unknown system states or model coefficients. Therefore, the proof of asymptotic convergence of the modified error $z$ is valid upon these controller features.

Remark 19. The size of the convergence region $\Omega_{z}$, that is $C_{b e}$, is user-defined so that it does not depend on the bounds of either external disturbances, system states, or model coefficients. Consequently, such bounds are not required by the formulated controller. This is a significant improvement with respect to current AES-based adaptive controller, e.g., [19,25].

Remark 20. In the case that input constraint were not taken into account, the controller design would be based on a dead zone function of the tracking error, $f_{e}$, featuring $f_{e}=0$ when the tracking error e is inside the convergence set $\Omega_{e}$, that is, $|e| \leq C_{b e}$. Moreover, the updated parameters would be integrals of $f_{e}$ terms. However, the convergence of $e$ and the boundedness of the updated parameters would not be guaranteed for input constraint. Indeed, during input saturation moments, the tracking error would increase and $e \notin \Omega_{e}$. Therefore, the updated parameters would continue increasing and excessive increase would occur. In contrast, in the case that input constraint is handled via AES strategy (Section 4): (i) the controller design is based on a dead zone function of the modified tracking error $\left(f_{z}\right)$, instead of $f_{e}$, where the modified tracking error is $z=y_{m}-y_{d}-\psi, \psi$ 
is a filter in terms of the input error, and $f_{z}=0$ when $z$ is inside the convergence set $\Omega_{z}$, that is, $|z| \leq C_{b e}$; (ii) the updated parameters are integrals of $f_{z}$ instead of $f_{e}$ terms; (iii) the function $f_{z}$ is guaranteed to converge to zero and $z$ is guaranteed to converge to $\Omega_{z}$ despite input saturation, as stated by Theorem 2. During input saturation events, the convergence of $f_{z}$ implies that the updated parameters do not experience excessive increase. In addition, the boundedness of updated parameter vectors $\hat{\theta}$ and $\hat{\theta}_{b}$ is proved by Lyapunov stability theory, in the proof of Theorem 1.

\section{Simulation Results}

Recall that the formulated controller corresponds to the mathematical model of the fed-batch submerged cultivation kinetics presented in Equation (1) to (3) subject to input constraint (4), Assumptions 1 to 5, being the control goal and the desired output $y_{d}$ stated in Section 3. The control and update laws and the corresponding parameters are stated in Remark 12. At what follows, the values of $S, S_{i m}, S_{m}$, and $V_{m}$ are generated by using the model (1) to (3) with specific parameter values, a specific form of $\mu$, and the measurement noise models (5) to (7) with specific values of measurement errors. These values of $S_{m}$, $S_{i m}$, and $V_{m}$ are used by the controller, but the $\mu$ expression, the model parameters, the specific values of measurement errors, and upper or lower bounds of model parameters or measurement errors are not.

We consider Gluconacetobacter diazotrophicus culture [67], and we assume that an inflow is incorporated thus resulting in a fed-batch process, so that model (1) to (3) applies. The model therein is taken to the form (1) to (3), so that $\mu=\mu_{\max } S /(B X+S)-k_{0}$. The model parameters are shown in Table 1.

Table 1. Model parameters.

\begin{tabular}{cc}
\hline Parameter & Value \\
\hline$Y$ & 7.716 \\
$B$ & 1496.2 \\
$S_{\text {in }}$ & $100 \mathrm{~g} / \mathrm{L}$ \\
$\mu_{\max }$ & $0.7058 \mathrm{~h}-1$ \\
$V_{t o}$ & $20 \mathrm{~L}$ \\
$k_{o}$ & $0.0179 \mathrm{~h}^{-1}$
\end{tabular}

Furthermore, the particular values of the closed loop starting time $t_{o}$ and the reference model parameters $\left(a_{m}, \bar{r}\right)$ correspond to this culture. The measurement errors are assumed as:

$$
\delta_{s i}=N_{s}, a_{o}=N_{s}, \delta_{v}=0.0028 N_{s}
$$

where $N_{s}$ is a filtered random signal in the range [ $\left.-0.20 .2\right]$.

A no feeding phase $\left(Q_{i n}>0\right)$ is used before the feeding phase, comprising substrate consumption and biomass growth. The feeding phase $\left(Q_{i n}>0\right)$ is started when $S_{m}$ reaches the critical value $S^{*}$. Recall that this strategy can be used for fed-batch bioreactors [4,34].

The $Q_{\text {in }}$ saturation values, the parameters of the reference model, the user defined parameters of the control and update laws, and the use of auxiliary system are defined according to three cases, whereas the desired width of the convergence set is chosen as $C_{b e}=0.4$ and $u_{a}=\left(u_{\min }+u_{\max }\right) / 2, S^{*}=20 \mathrm{~g} / \mathrm{L}$ and $t_{o}$ is the time when $S_{m}$ reaches the critical value $S^{*}$.

The three different cases aims at assessing the capability of the developed controller to achieve the expected performance under different values of the desired output $y_{d}$, the upper limit of the manipulated input $u_{\max }$, the user-defined parameters of the control law, and auxiliary system, $c_{1}, c_{2}$, the user-defined parameters of the update laws, $\gamma_{1}, \gamma_{2}, \gamma_{b}$ and the use of auxiliary system: 
(i). The $y_{d}$ trajectory is obtained through the reference model (18), the parameter $a_{m}$, and the signal $\bar{r}$ : in the first case, the $y_{d}$ trajectory is a step response of a low pass filter, ending in a constant value, whereas in the second and third cases it is a combination of that of the first case, with oscillations, in order to provide high values of $d y_{d} / d t$.

(ii). The $u_{\max }$ signal is higher in the second and third cases compared with the first case.

(iii). The user-defined parameters: in the second and third cases, the values of user-defined parameters are higher than those of the first case, in order to achieve a small tracking error despite the increased complexity of the $y_{d}$ signal.

(iv). The use of auxiliary system: in the third case, no auxiliary system is used.

\subsection{First Case}

The $Q_{\text {in }}$ saturation values are $u_{\min }=0, u_{\max }=0.0231 \mathrm{~L} / \mathrm{h}$. The parameters of the reference model are chosen as: $a_{m}=0.1$ and

$$
\bar{r}=\left\{\begin{array}{l}
S^{*} \text { for } t<t_{o} \\
30 \text { otherwise }
\end{array}\right.
$$

For the control and update laws we use $c_{1}=1, c_{2}=0.3, \gamma_{1}=0.1, \gamma_{2}=0.1, \gamma_{b}=0.01$.

Simulations show that the signals $z, e, S_{m}, u, v$ and the updated parameters $\hat{\theta}_{1}, \hat{\theta}_{2}$, $\hat{\theta}_{b}$ remain bounded (Figure 1 ). The modified tracking error $z$ is inside $\Omega_{z}$ at initial time, it is outside during [63.2 70.70] h, and it remains inside afterwards (Figure 1b). There is an input saturation event during the time lapse [65.40 70.89] h: (i) $z<-C_{b e}, e<-C_{b e}$ (Figure $1 \mathrm{~b}$ ) and the constrained signal $v$ saturates at its upper bound (Figure 1c); (ii) the updated parameters increase, but not excessively (Figure 1d), which is in accordance with Theorem 1. After that time period: (i) $v$ remains in a periodic behavior with no further saturation events, so that $\Delta u=0$ (Figure 1c); (ii) $z$ converges to $\Omega_{z}$ (Figure 1b), which is made possible by parameter updating undergone before; (iii) $e$ converges to $\Omega_{e}$ (Figure $1 b$ ), which is due to the convergence of $z$ to $\Omega_{z}$ and the ceasing of saturation events $(\Delta u=0)$ in accordance with Theorem 2; (iv) the updated parameters converge to some constant values, as a consequence of the convergence of $z$; in fact, they are constant when $z \in \Omega_{z}$ (Figure 1d).

\subsection{Second Case}

The $Q_{\text {in }}$ saturation values are $u_{\min }=0, u_{\max }=0.0385 \mathrm{~L} / \mathrm{h}$, whereas the parameters of the reference model are $a_{m}=1 / 5$ and

$$
\bar{r}=\left\{\begin{array}{c}
S^{*} \text { for } t<t_{o} \\
30+1.2 \sin \left(\frac{2 \pi t}{20}\right) \text { otherwise }
\end{array}\right.
$$

For the control and update laws we use: $u_{a}=0, c_{1}=4, c_{2}=3, \gamma_{1}=0.2, \gamma_{2}=0.2, \gamma_{b}=0.2$. Simulations show that the signals $e, S_{m}, z, u, v$ and the updated parameters $\hat{\theta}_{1}, \hat{\theta}_{2}, \hat{\theta}_{b}$ remain bounded (Figure 2), and the culture volume $V$ is non-decreasing (Figure 3 ). The modified tracking error $z$ is inside $\Omega_{z}$ at initial time, it is outside during [63.15 67.92] h, and it remains inside afterwards (Figure $2 \mathrm{~b}$ ). There is an input saturation event during the time lapse [63.69 69.06] h: (i) $z<0, e<-C_{b e}$ (Figure $2 \mathrm{~b}, \mathrm{f}$ ) and the constrained signal $v$ saturates at its upper bound (Figure 2c,e); (ii) the updated parameters increase, but not excessively (Figure 2d), which is in accordance with Theorem 1. 
(a)

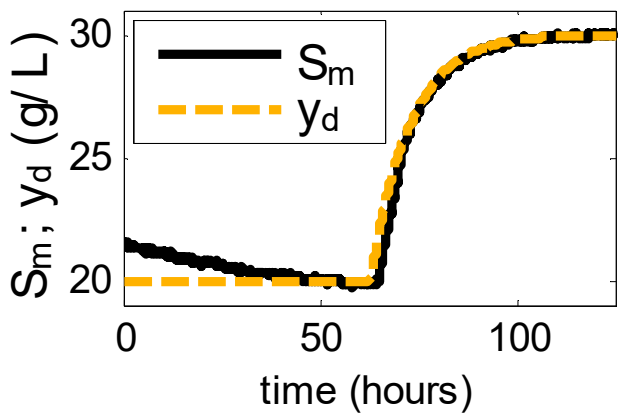

(c)

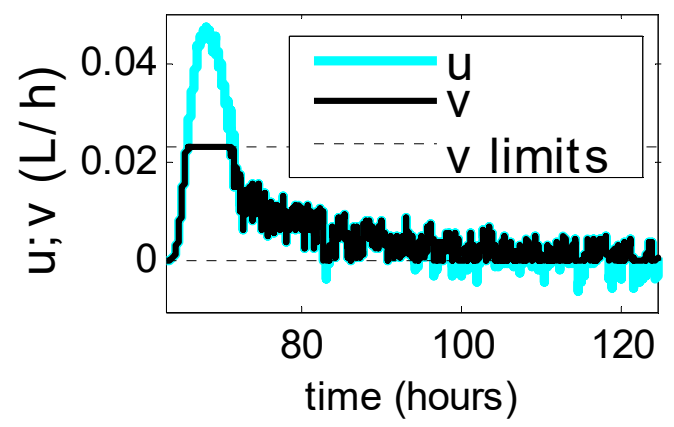

(e)

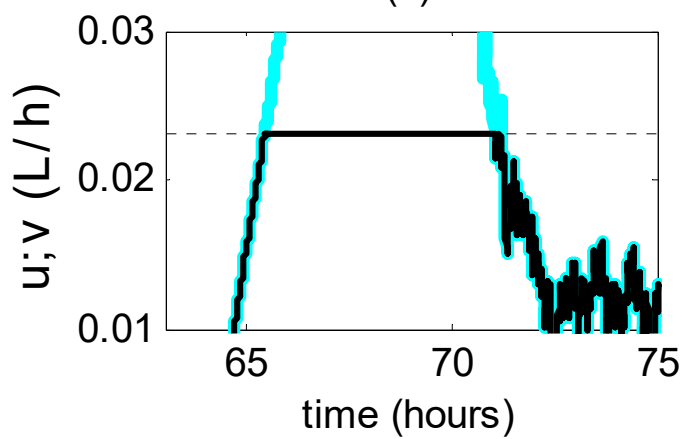

(b)

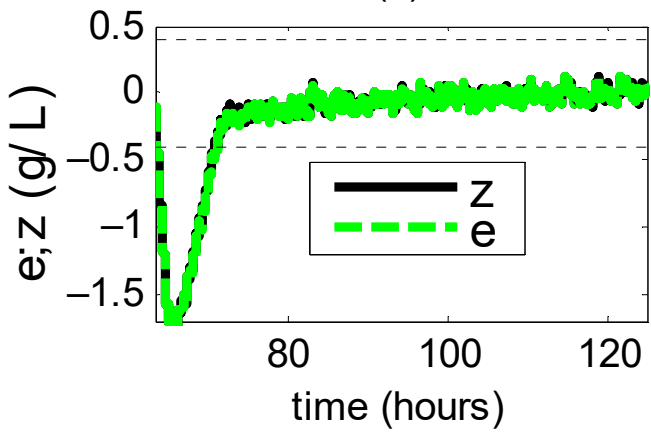

(d)

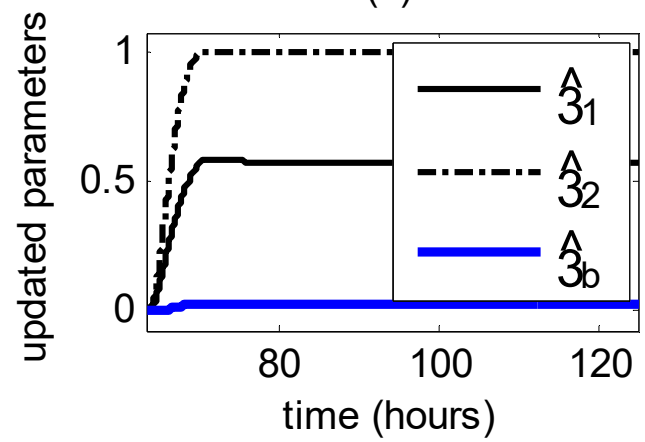

(f)

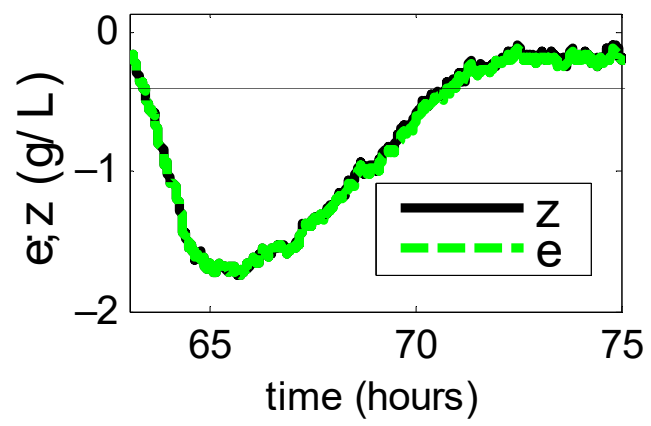

Figure 1. Simulation results for the first case of reference model and controller parameter values. (a) Time course of the measured output $S_{m}$ and the desired output $y_{d}$. (b) Time course of the tracking error $e$ and the modified error $z$. (c) Time course of the non-saturated input $u$ and the saturated input $v$. (d) Time course of the updated parameters $\hat{\theta}_{1}, \hat{\theta}_{2}, \hat{\theta}_{b}$. (e) Detail of non-saturated input $u$ and the saturated input $v$. (f) Detail of the tracking error $e$ and the modified error $z$. 
(a)

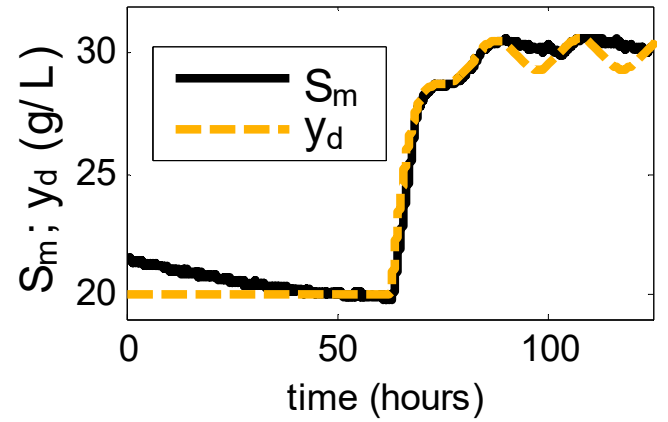

(c)

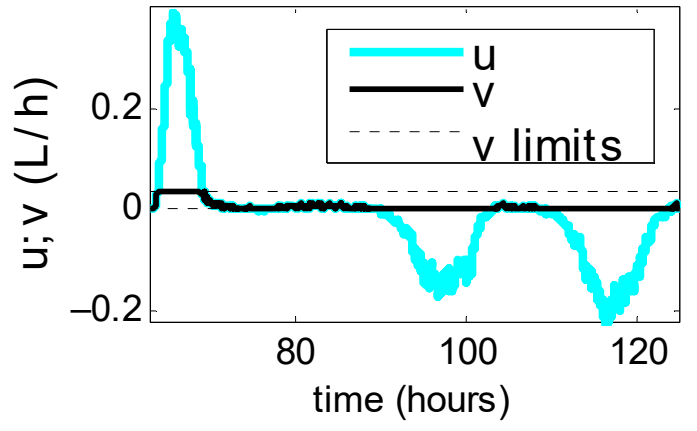

(e)

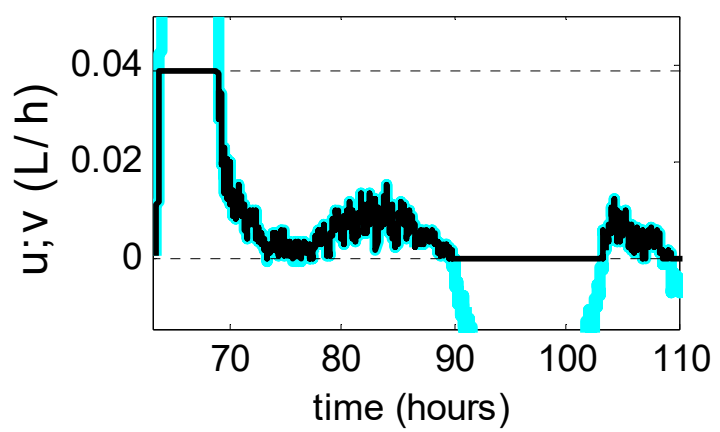

(b)

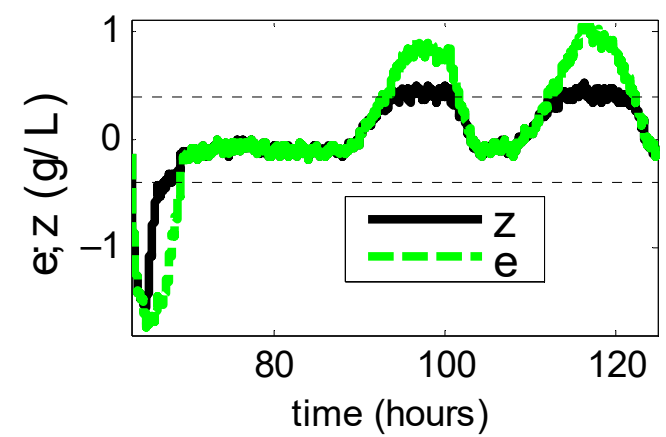

(d)

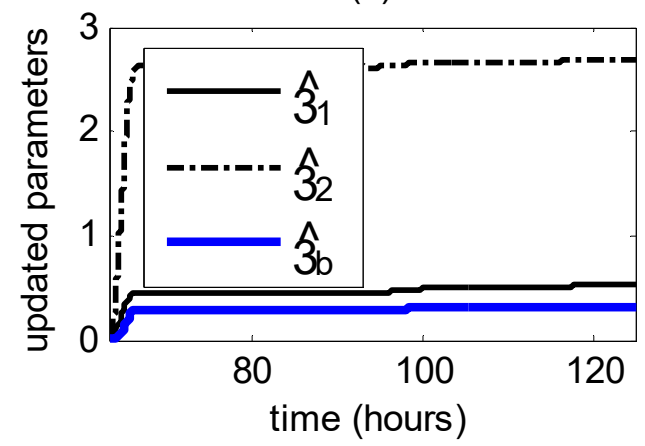

(f)

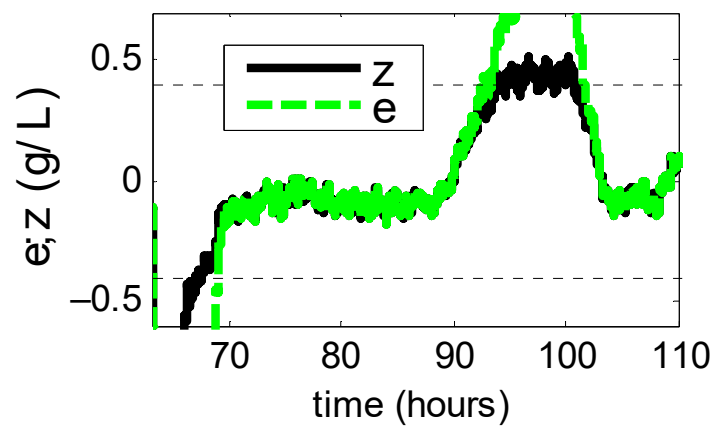

Figure 2. Simulation results for second case of reference model and controller parameter values. (a) Time course of the measured output $S_{m}$ and the desired output $y_{d}$. (b) Time course of the tracking error $e$ and the modified error $z$. (c) Time course of the non-saturated input $u$ and the saturated input $v$. (d) Time course of the updated parameters $\hat{\theta}_{1}, \hat{\theta}_{2}, \hat{\theta}_{b}$. (e) Detail of non-saturated input $u$ and the saturated input $v$. (f) Detail of the tracking error $e$ and the modified error $z$.

After that input saturation time period, $v$ remains in a periodic behavior with some further saturation events at its lower limit $\left(u_{\min }\right)$, (Figure $\left.2 \mathrm{c}, \mathrm{e}\right)$ and $z \in \Omega_{z}$ (Figure $2 \mathrm{~b}, \mathrm{f}$ ), which is made possible by parameter updating undergone before. Considering the whole closed loop regime, the tracking error $e$ does not converge to $\Omega_{e}$, which is due to the input saturation events. Indeed, $e \notin \Omega_{e}$ during input saturation events. However, it converges to a close neighborhood of $\Omega_{e}$, and $e \in \Omega_{e}$ during time lapses when saturation events cease, for instance during [101.92 112.5]h (Figure 2b,f), which is due to the convergence of $z$ to $\Omega_{z}$ and $\Delta u=0$ during these time lapses, according to Theorem 2 . The updated parameters remain constant during the time lapse featuring $z \in \Omega_{z}$, and remain unaffected by input saturation events (Figure $2 b, f)$. 
(a)

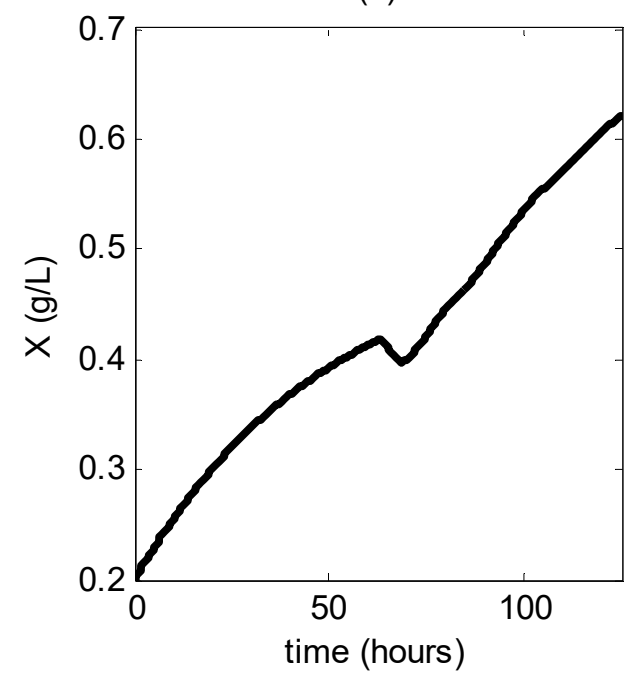

(b)

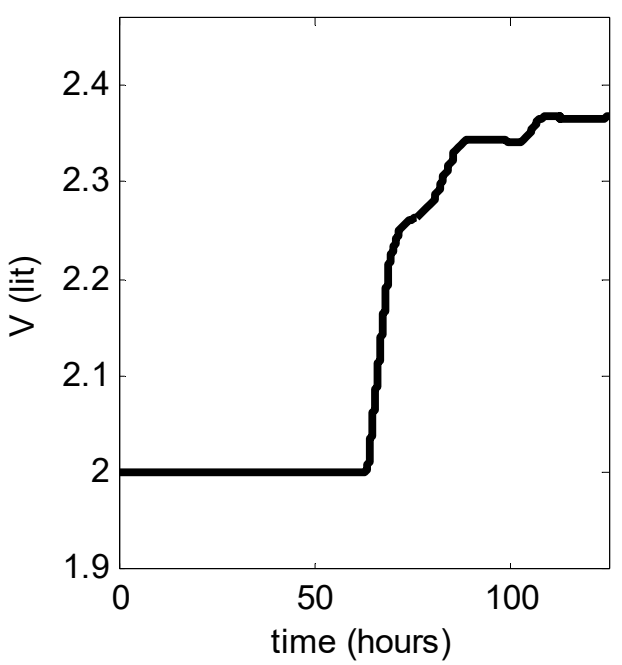

Figure 3. Simulation results for second case of reference model and controller parameter values. (a) Time course of the biomass concentration X. (b) Time course of the culture volume V.

\subsection{Third Case}

In the third case, the $Q_{i n}$ saturation values $\left(u_{\text {min }}, u_{\max }\right)$, the parameters of the reference model $\left(a_{m}, \bar{r}\right)$, the user-defined parameters of the control law and auxiliary system $\left(u_{a}, c_{1}\right.$, $\left.c_{2}\right)$, and the user-defined parameters of the update laws $\left(\gamma_{1}, \gamma_{2}\right)$ are the same as those of the second case. However, no auxiliary system is used, or equivalently, $\psi=0$, so that $\hat{\theta}_{b}$ is not necessary.

Simulations show that the signals $e, S_{m}, z, u, v$ remain bounded but not the updated parameters $\hat{\theta}_{1}, \hat{\theta}_{2}$ (Figure 4). The modified tracking error $z$ is inside $\Omega_{z}=\left\{z:|z| \leq C_{b e}\right\}$ at initial time, and it exhibits intermittent dwelling periods inside $\Omega_{\mathrm{z}}$ but it does not converge to $\Omega_{\mathrm{z}}$ (Figure $4 \mathrm{~b}$ ). There are several input saturation events after $89 \mathrm{~h}$, during which: (i) $z>C_{b e}, e>C_{b e}$ (Figure $4 \mathrm{~b}, \mathrm{f}$ ); (ii) the constrained signal $v$ saturates at its lower bound (Figure $4 \mathrm{c}, \mathrm{e}$ ); (iii) the updated parameters increase as $z>C_{b e}$, with no upper bound (Figure $4 \mathrm{~d}$ ). This simulation indicates us the importance of taking into account the effect of input saturation, and the effectivity of the improved auxiliary system in avoiding excessive increase of updated parameters.

\subsection{General Discussion on the Simulation Results}

The simulations results verify that the developed controller achieves satisfactory convergence of the modified error $z$ to the predefined compact set $\Omega_{z}=\left\{z:|z| \leq C_{b e}\right\}$, with a small steady value and a small settling time, but also: the lack of knowledge on the exact values and bounds of the control gain and model terms is tackled by means of updated parameters, such that proper convergence of the signal $z$ is achieved despite this uncertainty; the effect of input saturation is tackled by means of the auxiliary system, such that convergence of the signal $z$ is achieved; discontinuous signals are used in neither the control law, nor the update law nor the auxiliary system, and saturation type signals are used instead, whereas the convergence of the signal $z$ is not affected by this fact; excessive increase of updated parameters is avoided; the tracking error $e$ converges to the user-defined residual set $\Omega_{e}=\left\{e:|e| \leq C_{b e}\right\}$ during periods with no input saturation.

The simulations show that the updated parameters remain constant when $z$ is inside its compact set $\Omega_{z}$, which verifies the effect of signal $z$ in the update laws. 
(a)

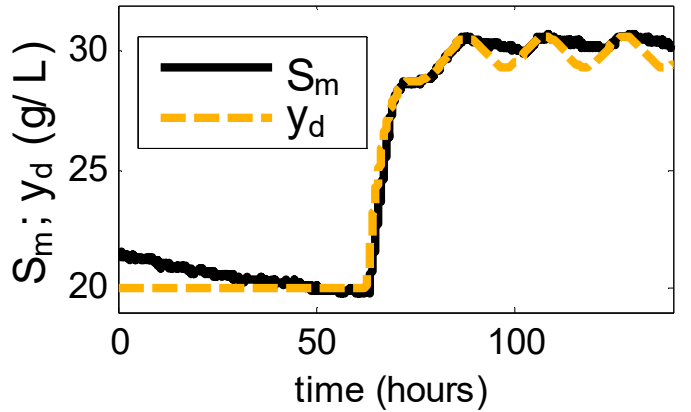

(c)

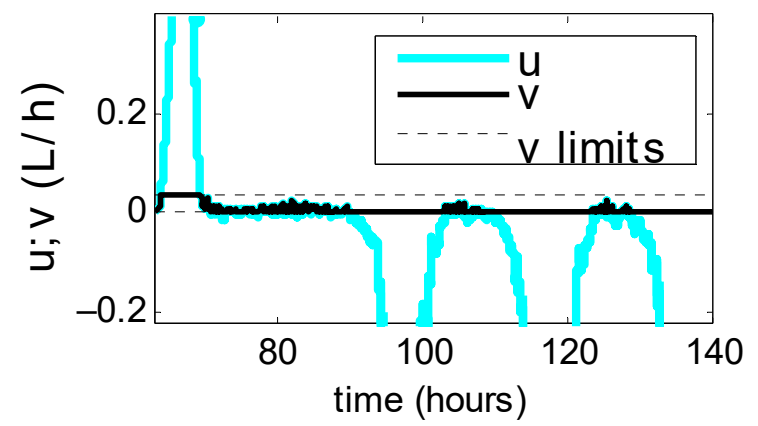

(e)

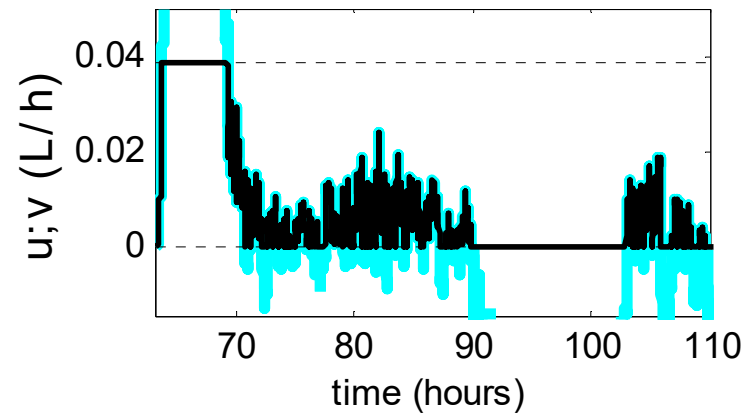

(b)

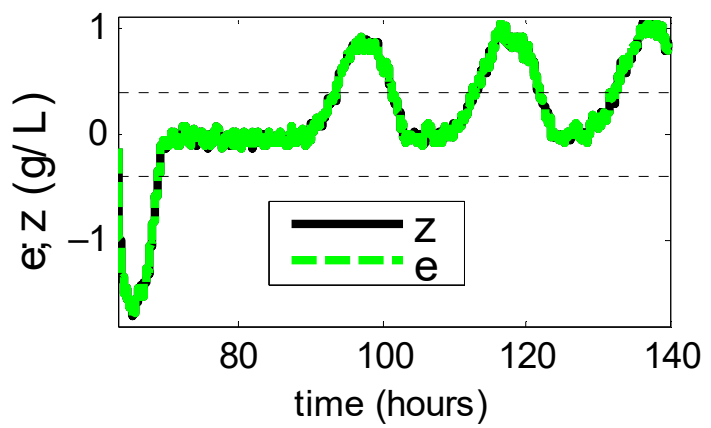

(d)

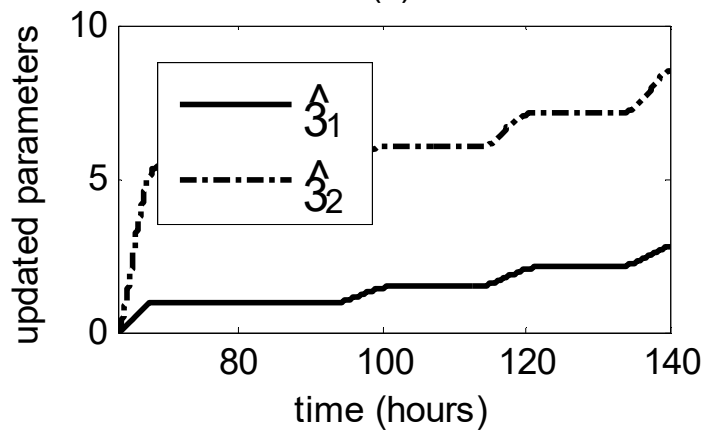

(f)

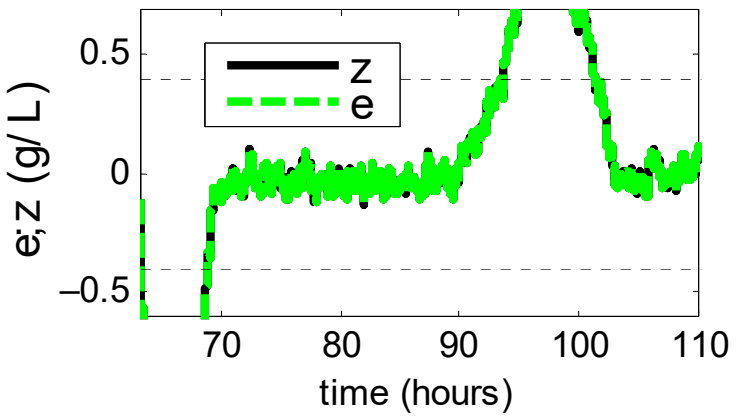

Figure 4. Simulation results for the third simulation case. (a) Time course of the measured output $S_{m}$ and the desired output $y_{d}$. (b) Time course of the tracking error $e$ and the modified error $z$. (c) Time course of the non-saturated input $u$ and the saturated input $v$. (d) Time course of the updated parameters $\hat{\theta}_{1}, \hat{\theta}_{2}$. (e) Detail of non-saturated input $u$ and the saturated input $v$. (f) Detail of the tracking error $e$ and the modified error $z$.

From the simulations during the oscillatory behavior of $y_{d}$ in the second case for $t \geq 63.2 \mathrm{~h}$ it follows that: (i) excessive increase of the updated parameters is avoided despite reiterated saturation events, during which the tracking error $e$ remains outside the compact set $\Omega_{e}$; (ii) the tracking error $e$ converges to $\Omega_{e}$ during periods with no input saturation $(\Delta u=0)$, but it is outside $\Omega_{e}$ during periods with input saturation $(\Delta u \neq 0)$. In addition, the oscillations of $y_{d}$ imply that a higher control effort is required in order to achieve the convergence of the output $y$ to $y_{d}$. To this end, higher values of controller parameters $c_{1}, c_{2}, \gamma_{1}, \gamma_{2}, \gamma_{b}$ have been used in the second case. However, the limitation of the control effort due to input saturation leads to limited speed of the output $y$, so that it fails to reach $y_{d}$, and the values of the tracking error $|e|$ are higher than required, although only during moments of higher $d y_{d} / d t$ values.

\section{Conclusions}

In this paper, a new adaptive controller was formulated for a fed-batch culture subject to output measurement noise, unknown parameter variation, input saturation, and unknown varying control gain with unknown lower and upper bounds. The proposed 
procedure is novel with respect to that of controller designs aimed at tackling input saturation. The rewriting of the $b \Delta u$ term, the formulation of the auxiliary system and the parameterization of the terms related to model parameters are novel tasks. It was ensured that: (i) Excessive increase of updated parameters is avoided despite input saturation; (ii) the tracking error converges to a compact set whose width is user-defined, so that model coefficients, bounds on external disturbances and model terms are not required to be known; (iii) the modified tracking error and the regular tracking error are bounded despite input saturation. In addition, saturation functions of the modified tracking error are used instead of discontinuous signum type functions, so as to avoid undesired chattering. To this end, the design is based on quadratic forms with vertex dead zone. The proposed approach can be applied to other SISO nonlinear continuous time systems.

Author Contributions: Conceptualization, A.R.; methodology, A.R.; writing-original draft preparation, A.R.; writing-review and editing, G.M.R. and Ó.J.S.; visualization, G.M.R. and Ó.J.S. All authors have read and agreed to the published version of the manuscript.

Funding: A.R. and G.M.R. were supported by Universidad Católica de Manizales.

Data Availability Statement: Not applicable.

Conflicts of Interest: The authors declare no conflict of interest.

\section{References}

1. Jamilis, M.; Garelli, F.; De Battista, H. Growth rate maximization in fed-batch processes using high order sliding controllers and observers based on cell density measurement. J. Process Control 2018, 68, 23-33. [CrossRef]

2. Nuñez, S.; Garelli, F.; De Battista, H. Closed-loop growth-rate regulation in fed-batch dual-substrate processes with additive kinetics based on biomass concentration measurement. J. Process Control 2016, 44, 14-22. [CrossRef]

3. Mailleret, L.; Bernard, O.; Steyer, J.-P. Nonlinear adaptive control for bioreactors with unknown kinetics. Automatica 2004, 40, 1379-1385. [CrossRef]

4. Van Impe, J.; Bastin, G. Optimal adaptive control of fed-batch fermentation processes with multiple substrates. Proc. IEEE Int. Conf. Control. Appl. 2002, 3, 469-474. [CrossRef]

5. De Battista, H.; Jamilis, M.; Garelli, F. Global stabilisation of continuous bioreactors: Tools for analysis and design of feeding laws. Automatica 2018, 89, 340-348. [CrossRef]

6. De Battista, H.; Picó, J.; Picó-Marco, E. Nonlinear PI control of fed-batch processes for growth rate regulation. J. Process Control 2012, 22, 789-797. [CrossRef]

7. Åkesson, M.; Hagander, P.; Axelsson, J.P. Probing control of fed-batch cultivations: Analysis and tuning. Control. Eng. Pract. 2001, 9, 709-723. [CrossRef]

8. Axelsson, J. On the Role of Adaptive Controllers in Fed-Batch Yeast Production. IFAC Proc. Vol. 1988, 21, 103-108. [CrossRef]

9. Hagander, P.; Axelsson, J.P.; Holst, O. Substrate control of biotechnical fedbatch processes robustness and the role of adaptivity. IFAC Proc. Vol. 1990, 23, 253-258. [CrossRef]

10. Smets, I.; Bastin, G.; Van Impe, J. Feedback Stabilization of Fed-Batch Bioreactors: Non-Monotonic Growth Kinetics. Biotechnol. Prog. 2002, 18, 1116-1125. [CrossRef]

11. Mears, L.; Stocks, S.M.; Sin, G.; Gernaey, K.V. A review of control strategies for manipulating the feed rate in fed-batch fermentation processes. J. Biotechnol. 2017, 245, 34-46. [CrossRef]

12. Vargas, A.; Moreno, J.; Wouwer, A.V. Super-twisting estimation of a virtual output for extremum-seeking output feedback control of bioreactors. J. Process Control 2015, 35, 41-49. [CrossRef]

13. Petre, E.; Selisteanu, D.; Roman, M. Nonlinear robust adaptive control strategies for a lactic fermentation process. J. Chem. Technol. Biotechnol. 2018, 93, 518-526. [CrossRef]

14. Rincón, A.; Piarpuzán, D.; Angulo, F. A new adaptive controller for bio-reactors with unknown kinetics and biomass concentration: Guarantees for the boundedness and convergence properties. Math. Comput. Simul. 2015, 112, 1-13. [CrossRef]

15. Petre, E.; Selişteanu, D.; Şendrescu, D. Adaptive and robust-adaptive control strategies for anaerobic wastewater treatment bioprocesses. Chem. Eng. J. 2013, 217, 363-378. [CrossRef]

16. Lara-Cisneros, G.; Femat, R.; Dochain, D. An extremum seeking approach via variable-structure control for fed-batch bioreactors with uncertain growth rate. J. Process Control 2014, 24, 663-671. [CrossRef]

17. Priyanka; Roy, S.; Chopda, V.; Gomes, J.; Rathore, A.S. Piyanka Comparison and implementation of different control strategies for improving production of rHSA using Pichia pastoris. J. Biotechnol. 2018, 290, 33-43. [CrossRef] [PubMed]

18. He, W.; Dong, Y.; Sun, C. Adaptive Neural Impedance Control of a Robotic Manipulator with Input Saturation. IEEE Trans. Syst. Man Cybern. Syst. 2016, 46, 334-344. [CrossRef]

19. Gao, S.; Ning, B.; Dong, H. Fuzzy dynamic surface control for uncertain nonlinear systems under input saturation via truncated adaptation approach. Fuzzy Sets Syst. 2016, 290, 100-117. [CrossRef] 
20. Hosseinzadeh, M.; Yazdanpanah, M.J. Robust adaptive passivity-based control of open-loop unstable affine non-linear systems subject to actuator saturation. IET Control. Theory Appl. 2017, 11, 2731-2742. [CrossRef]

21. Yang, Y.; Tan, J.; Yue, D. Prescribed Performance Tracking Control of a Class of Uncertain Pure-Feedback Nonlinear Systems with Input Saturation. IEEE Trans. Syst. Man Cybern. Syst. 2018, 50, 1733-1745. [CrossRef]

22. Wang, Q.; Duan, G.-R. Output feedback stabilization of spacecraft autonomous rendezvous subject to actuator saturation. Int. J. Autom. Comput. 2016, 13, 428-437. [CrossRef]

23. Zhang, X.; Li, X.; Zhao, J. Stability analysis and anti-windup design of switched systems with actuator saturation. Int. J. Control. Autom Syst. 2018, 16, 1247-1253. [CrossRef]

24. Polycarpou, M.; Farrell, J.; Sharma, M. On-line approximation control of uncertain nonlinear systems: Issues with control input saturation. In Proceedings of the American Control Conference 2003, Denver, CO, USA, 4-6 June 2003. [CrossRef]

25. Min, H.; Xu, S.; Ma, Q.; Zhang, B.; Zhang, Z. Composite-observer-based output-feedback control for nonlinear time-delay systems with input saturation and its application. IEEE Trans. Ind Electron. 2018, 65, 5856-5863. [CrossRef]

26. Lin, D.; Wang, X.; Yao, Y. Fuzzy neural adaptive tracking control of unknown chaotic systems with input saturation. Nonlinear Dyn. 2011, 67, 2889-2897. [CrossRef]

27. Gao, S.; Dong, H.; Ning, B.; Yao, X. Single-parameter-learning-based fuzzy fault-tolerant output feedback dynamic surface control of constrained-input nonlinear systems. Inf. Sci. 2017, 385-386, 378-394. [CrossRef]

28. Li, Y.; Tong, S.; Li, T. Adaptive fuzzy output-feedback control for output constrained nonlinear systems in the presence of input saturation. Fuzzy Sets Syst. 2014, 248, 138-155. [CrossRef]

29. Nassira, Z.; Mohamed, C.; Essounbouli, N. Adaptive neural-network output feedback control design for uncertain CSTR system with input saturation. In Proceedings of the 2018 International Conference on Electrical Sciences and Technologies in Maghreb (CISTEM) 2019, Algiers, Algeria, 28-31 October 2018. [CrossRef]

30. Askari, M.R.; Shahrokhi, M.; Talkhoncheh, M.K. Observer-based adaptive fuzzy controller for nonlinear systems with unknown control directions and input saturation. Fuzzy Sets Syst. 2017, 314, 24-45. [CrossRef]

31. Mutturi, S. Dynamic optimization of fed-batch bioprocesses using flower pollination algorithm. Bioprocess. Biosyst. Eng. 2018, 41, 1679-1696. [CrossRef] [PubMed]

32. Mohseni, S.S.; Babaeipour, V.; Vali, A.R. Design of sliding mode controller for the optimal control of fed-batch cultivation of recombinant E. coli. Chem. Eng. Sci. 2009, 64, 4433-4441. [CrossRef]

33. Chen, L.Z.; Chen, X.D.; Nguang, S.K. Designing and implementing optimal control of fed-batch fermentation processes. In Modelling and Optimization of Biotechnological Processes; Chen, L.Z., Chen, X.D., Nguang, S.K., Eds.; Springer: Berlin/Heidelberg, Germany, 2006; pp. 91-108.

34. Birol, G.; Ündey, C.; Cinar, A. A modular simulation package for fed-batch fermentation: Penicillin production. Comp. Chem. Eng. 2002, 26, 1553-1565. [CrossRef]

35. Çakir, T.; Arga, K.Y.; Altintas, M.M.; Ülgen, K.Ö. Flux analysis of recombinant Saccharomyces cerevisiae YPB-G utilizing starch for optimal ethanol production. Process Biochem. 2004, 39, 2097-2108. [CrossRef]

36. Cardona, C.A.; Sánchez, O.J.; Gutiérrez, L.F. Process Synthesis for Fuel Ethanol Production; CRC Press: Boca Ratón, FL, USA, 2010.

37. Markana, A.; Padhiyar, N.; Moudgalya, K. Multi-criterion control of a bioprocess in fed-batch reactor using EKF based economic model predictive control. Chem. Eng. Res. Des. 2018, 136, 282-294. [CrossRef]

38. Bastin, G.; Dochain, D. On-Line Estimation and Adaptive Control of Bioreactors; Elsevier: Amsterdam, The Netherlands, 1990.

39. Méndez-Acosta, H.O.; Campos-Delgado, D.U.; Femat, R.; González-Alvarez, V. A robust feedforward/feedback control for an anaerobic digester. Comput. Chem. Eng. 2005, 29, 1613-1623. [CrossRef]

40. Chang, L.; Liu, X.; Henson, M.A. Nonlinear model predictive control of fed-batch fermentations using dynamic flux balance models. J. Process Control 2016, 42, 137-149. [CrossRef]

41. Hafidi, G.; Tebbani, S.; Dumur, D.; Vande Woumer, A. Robustness analysis of nonlinear predictive control of fed-batch E. coli cultures. In Proceedings of the 16th Mediterranean Conference on Control and Automation, Ajaccio, France, $25-27$ June 2008.

42. Jin, H.; Zheng, Z.; Gao, M.; Duan, D.; Shi, Z.; Wang, Z.; Jin, J. Effective induction of phytase in Pichia pastoris fed-batch culture using an ANN pattern recognition model-based on-line adaptive control strategy. Biochem. Eng. J. 2007, 37, 26-33. [CrossRef]

43. Cougnon, P.; Dochain, D.; Guay, M.; Perrier, M. On-line optimization of fedbatch bioreactors by adaptive extremum seeking control. J. Process Control 2011, 21, 1526-1532. [CrossRef]

44. De Battista, H.; Picó, J.; Picó-Marco, E. Globally stabilizing control of fed-batch processes with Haldane kinetics using growth rate estimation feedback. J. Process Control 2006, 16, 865-875. [CrossRef]

45. Picó-Marco, E.; Picó, J.; De Battista, H. Sliding mode scheme for adaptive specific growth rate control in biotechnological fed-batch processes. Int. J. Control. 2005, 78, 128-141. [CrossRef]

46. Yao, Y. Dynamics of a delay turbidostat system with contois growth rate. Math. Biosci. Eng. 2019, 16, 56-77. [CrossRef]

47. Sari, T.; Mazenc, F. Global dynamics of the chemostat with different removal rates and variable yields. Math. Biosci. Eng. 2011, 8, 827-840. [CrossRef]

48. Hfaïedh, K.; Dahech, K.; Damak, T. A sliding mode observer for uncertain nonlinear systems based on multiple models approach. Int. J. Autom. Comput. 2016, 14, 202-212. [CrossRef]

49. Kole, M.M.; Draper, I.; Gerson, D.F. Production of protease by Bacillus subtilis using simultaneous control of glucose and ammonium concentrations. J. Chem. Technol. Biotechnol. 2007, 41, 197-206. [CrossRef] 
50. Wang, G.; Post, W.M. A theoretical reassessment of microbial maintenance and implications for microbial ecology modeling. FEMS Microbiol. Ecol. 2012, 81, 610-617. [CrossRef]

51. Zhang, D.; Dechatiwongse, P.; del-rio-Chanona, E.A.; Hellgardt, K.; Maitland, G.C.; Vassiliadis, V.S. Analysis of the cyanobacterial hydrogen photoproduction process via model identification and process simulation. Chem. Eng. Sci. 2015, 128, 130-146. [CrossRef]

52. García-Diéguez, C.; Molina, F.; Roca, E. Multi-objective cascade controller for an anaerobic digester. Process. Biochem. 2011, 46, 900-909. [CrossRef]

53. Picó, J.; De Battista, H.; Garelli, F. Smooth sliding-mode observers for specific growth rate and substrate from biomass measurement. J. Process Control 2009, 19, 1314-1323. [CrossRef]

54. Méndez-Acosta, H.O.; Palacios-Ruiz, B.; Alcaraz-González, V.; González-Álvarez, V.; García-Sandoval, J.P. A robust control scheme to improve the stability of anaerobic digestion processes. J. Process Control 2010, 20, 375-383. [CrossRef]

55. De Battista, H.; Picó, J.; Garelli, F.; Vignoni, A. Specific growth rate estimation in (fed-)batch bioreactors using second-order sliding observers. J. Process Control 2011, 21, 1049-1055. [CrossRef]

56. Méndez-Acosta, H.O.; Palacios-Ruiz, B.; Alcaraz-González, V.; Steyer, J.P.; González-Álvarez, V.; Latrille, E. Robust control of volatile fatty acids in anaerobic digestion processes. Ind. Eng. Chem. Res. 2008, 47, 7715-7720. [CrossRef]

57. Ignatova, M.N.; Lyubenova, V.N.; García, M.R.; Vilas, C.; Alonso, A.A. Indirect adaptive linearizing control of a class of bioprocesses-estimator tuning procedure. J. Process Control 2008, 18, 27-35. [CrossRef]

58. López Pérez, P.A.; Neria González, M.I.; Pérez García, M.R.; Aguilar López, R. Concentrations monitoring via software sensor for bioreactors under model parametric uncertainty: Application to cadmium removal in an anaerobic process. Alex. Eng. J. 2016, 55, 1893-1902. [CrossRef]

59. Polycarpou, M.M.; Ioannou, P.A. A robust adaptive nonlinear control design. Automatica 1996, 32, 423-427. [CrossRef]

60. Polycarpou, M.M.; Ioannou, P.A. On the existence and uniqueness of solutions in adaptive control systems. IEEE Trans. Automat. Contr. 1993, 38, 474-479. [CrossRef]

61. Slotine, J.; Li, W. Applied Nonlinear Control; Prentice-Hall Inc.: Englewood Cliffs, NJ, USA, 1991.

62. Koo, K. Stable adaptive fuzzy controller with time-varying dead-zone. Fuzzy Sets Syst. 2001, 121, 161-168. [CrossRef]

63. Zhou, J.; Zhang, C.; Wen, C. Robust adaptive output control of uncertain nonlinear plants with unknown backlas nonlinearity. IEEE Trans. Automat. Contr. 2007, 52, 503-509. [CrossRef]

64. Su, C.; Feng, Y.; Hong, H.; Chen, X. Adaptive control of system involving complex hysteretic nonlinearities: A generalised Prandtl-Ishlinskii modelling approach. Int. J. Control. 2009, 82, 1786-1793. [CrossRef]

65. Ranjbar, E.; Yaghubi, M.; Suratgar, A.A. Robust adaptive sliding mode control of a MEMS tunable capacitor based on dead-zone method. Automatika 2020, 61, 587-601. [CrossRef]

66. Ioannou, P.; Sun, J. Robust Adaptive Control; Prentice-Hall: PTR Upper Saddle River, NJ, USA, 1996.

67. Rincón, A.; Cuellar, J.A.; Valencia, L.F.; Sánchez, O.J. Cinética de crecimiento de Gluconacetobacter diazotrophicus usando melaza de caña y sacarosa: Evaluación de modelos cinéticos. Acta Biológica Colomb. 2019, 24, 38-57. [CrossRef] 